Homology, Homotopy and Applications, vol.6(1), 2004, pp.201-236

\title{
OPERATIONS AND CO-OPERATIONS IN MORAVA E-THEORY
}

\author{
MARK HOVEY \\ (communicated by Andrew Baker)
}

\begin{abstract}
Let $E=E_{n}$ denote the Morava $E$-theory spectrum, and let $\Gamma$ be the Morava stabilizer group of ring spectrum isomorphisms of $E$. We revisit the isomorphism $\pi_{*} L_{K(n)}(E \wedge E) \cong$ $C\left(\Gamma, E_{*}\right)$ of graded formal Hopf algebroids, and its dual isomorphism $E^{*} E \cong E_{*}[[\Gamma]]$.
\end{abstract}

\section{Introduction}

Let $p$ denote an integer prime, $n$ denote a nonnegative integer, and let $E$ denote the Morava $E$-theory spectrum $E_{p, n}$. This is the Landweber exact ring spectrum with

$$
E_{*} \cong W \mathbb{F}_{p^{n}}\left[\left[u_{1}, \ldots, u_{n-1}\right]\right]\left[u, u^{-1}\right],
$$

where the degree of $u_{i}$ is 0 for all $i$ and the degree of $u$ is 2 . There is an algebra map $B P_{*} \rightarrow E_{*}$ that takes $v_{i}$ to $u_{i} u^{p^{i}-1}$ for $i<n$, takes $v_{n}$ to $u^{p^{n}-1}$, and takes $v_{i}$ to 0 for $i>n$. Morava $E$-theory is closely related to the Johnson-Wilson theory $E(n)$; in fact $E$ is a finite free module over the localization $L_{K(n)} E(n)$ of $E(n)$ with respect to Morava $K$-theory $K(n)$.

The Morava $E$-theory spectrum $E$ is very important in algebraic topology. It is local with respect to $K(n)$, and plays a major role in the structure of the $K(n)$-local homotopy category [HS99]. Let $\Gamma$ denote the group of ring spectrum automorphisms (in the stable homotopy category) of $E$. Then $\Gamma$ is a version of the Morava stabilizer group [Str00, Proposition 4], and the famous result of Hopkins-Miller [Rez98] says that $E$ is in fact an $A_{\infty}$-ring spectrum and that $\Gamma$ is isomorphic to the group of components of the space of $A_{\infty}$ self-maps of $E$.

It is then natural to compute the operations $E^{*} E$ and co-operations $E_{*} E$ of $E$. Because $E$ is $K(n)$-local, it is more natural to look at $E_{*}^{\vee} E=\pi_{*} L_{K(n)}(E \wedge E)$ rather than the actual co-operations. This ring turns out to be the completion of $E_{*} E$ at the maximal ideal $\mathfrak{m}$. The answer has then been known to the experts for quite some time; $E_{*}^{\vee} E$ is isomorphic to $C\left(\Gamma, E_{*}\right)$, the ring of continuous functions from the profinite group $\Gamma$ to $E_{*}$ with its $\mathfrak{m}$-adic topology, where $\mathfrak{m}=\left(p, u_{1}, \ldots, u_{n-1}\right)$ is the unique homogeneous maximal ideal. Also, $E^{*} E$ is isomorphic to the completed twisted group ring $E_{*}[[\Gamma]]$.

Received December 19, 2003, revised April 22, 2004; published on May 17, 2004

2000 Mathematics Subject Classification: 55N22, 55P42, $57 \mathrm{~T} 05$.

Key words and phrases: Morava E-theory, Hopf algebroid, Morava stabilizer group, cohomology operations, twisted completed group ring.

(C) 2004, Mark Hovey. Permission to copy for private use granted. 
The first statement of this result seems to have been in an unpublished preprint of Hopkins and Ravenel [HR89], building on a similar statement for $K(n)$ in Morava's seminal paper [Mor85]. The first published proof of the co-operation result is due to Baker in [Bak95]; see also [Bak89]. A very short treatment is given in [DH04, Proposition 2.2], and a spectrum version of the isomorphism $E^{*} E \cong E_{*}[[\Gamma]]$ appears in Proposition 2.4 of [GHMR03]. To this author's eye, at least, these proofs are condensed and hard to follow. The general assumption is that Morava proved the result for $K(n)$ in [Mor85], and it is just a matter of getting efficiently from what Morava states to the actual result, by lifting using Hensel's lemma. Strickland approaches the result from a new perspective in [Str00, Theorem 12], as does K. Johnson in [Joh00]. But the calculation of $E_{*}^{\vee} E$ is really a side issue in both papers, so also gets relatively short shrift. There is also an unpublished preprint of Daniel Davis [Dav04], devoted to the theory of discrete $\Gamma$-spectra, that addresses the computation of $E_{*}^{\vee} E$.

In this paper, the author has tried to present the calculation of $E_{*}^{\vee} E$ in a fairly self-contained and conceptual way. Essentially, he has written a proof that he himself can understand, in the hope that this will also be useful to others. There are, after all, a great many details to be worked out. The basic idea, following Morava, is that $E_{*}^{\vee} E$ and $C\left(\Gamma, E_{*}\right)$ should represent the same functor on the category of rings. However, it is not at all obvious that this is the case. The first reduction is to divide by the maximal ideal $\mathfrak{m}$, and to eliminate the grading, reducing us to showing that $K_{0} E$ and $C\left(\Gamma, \mathbb{F}_{p^{n}}\right)$ represent the same functor. Even after identifying $\Gamma$ as a profinite topological group with an appropriate version of the Morava stabilizer group, which itself requires some nontrivial theory of Landweber exact spectra and profinite groups, this is still not clear. What is fairly simple to check is that $K_{0} E$ and $C\left(\Gamma, \mathbb{F}_{p^{n}}\right)$ have the same $\overline{\mathbb{F}_{p}}$-valued points, where $\overline{\mathbb{F}_{p}}$ is the algebraic closure of $\mathbb{F}_{p}$. The we use the fact that both algebras are ind-étale over $\mathbb{F}_{p^{n}}$ to complete the proof.

Here is an outline of the paper. Following Strickland [Str00], we first define the map

$$
\Phi: E_{*}^{\vee} E \rightarrow C\left(\Gamma, E_{*}\right)
$$

in Section 1. We also show that $\Gamma$ is profinite and acts continuously on $E_{*}$. We then prove, again following Strickland's proof, that both $E_{*}^{\vee} E$ and $C\left(\Gamma, E_{*}\right)$ are pro-free $E_{*}$-modules in Section 2. It follows that to prove $\Phi$ is an isomorphism, it suffices to prove that the map

$$
\Psi: K_{0} E \rightarrow C\left(\Gamma, \mathbb{F}_{p^{n}}\right)
$$

is an isomorphism, where $K=E / \mathfrak{m}$ is a variant on Morava $K$-theory. At this point, our proof diverges from Strickland's, as we now introduce the notion of ind-étale algebras in Section 3. We prove that both $K_{0} E$ and $C\left(\Gamma, \mathbb{F}_{p^{n}}\right)$ are ind-étale $\mathbb{F}_{p^{n-}}$ algebras. It follows that $\Psi$ is an isomorphism if and only if $\operatorname{Alg}_{\mathbb{F}_{p} n}\left(\Psi, \overline{\mathbb{F}_{p}}\right)$ is an isomorphism, where $\overline{\mathbb{F}_{p}}$ denotes the algebraic closure of $\mathbb{F}_{p}$. We then prove that $\operatorname{Alg}_{\mathbb{F}_{p^{n}}}\left(\Psi, \overline{\mathbb{F}_{p}}\right)$ is an isomorphism in Section 4, completing the proof that $\Phi$ is an isomorphism. This requires identification of the group $\Gamma$, also done by Strickland in $[\mathbf{S t r 0 0}]$ but with many details left out. 
Having completed the proof that $\Phi$ is an isomorphism in Theorem 4.11, we turn to cohomology operations in Section 5. We define the twisted completed group ring $E_{*}[[\Gamma]]$ and show that the natural map

$$
E_{*}[[\Gamma]] \rightarrow E^{*} E
$$

induced by the inclusion of $\Gamma$ in $E^{0} E$ is an isomorphism. We turn to Hopf algebroid structure in Section 6, defining the notion of a graded formal Hopf algebroid, showing that both $E_{*}^{\vee} E$ and $C\left(\Gamma, E_{*}\right)$ carry such structure, and proving that $\Phi$ preserves it. We close the paper with an appendix where we show that the topology on $\Gamma$ is entirely determined by its group structure. This is also proved in [Str00], but again with details left out.

The author would like to thank Neil Strickland, whose paper [Str00] was the inspiration for this one. It was also Strickland who first suggested the word "étale" to the author, which turned out to be the key idea to making the proof more conceptual. He would also like to thank Daniel Davis for his careful reading of the paper and his encouragement.

\section{The map $\Phi$}

In this section we define the map

$$
\Phi: E_{*}^{\vee} E \rightarrow C\left(\Gamma, E_{*}\right),
$$

and we show that $\Gamma$ is a first countable profinite group. This will require use of the natural topology, studied in [HS99, Section 11], on morphisms in the $K(n)$ local category. This natural topology is not very complicated, however, and we will summarize the properties of it that we need. Also, we will frequently use the notation $X \widetilde{\wedge} Y$ to mean $L_{K(n)}(X \wedge Y)$. With this notation, $E_{*}^{\vee} X=\pi_{*}(E \widetilde{\wedge} X)$.

Our construction of $\Phi$ will follow Strickland [Str00, Theorem 12]. As mentioned above, there is a natural topology on $[X, Y]$, for $X, Y K(n)$-local spectra [HS99, Section 11] (or objects in any algebraic stable homotopy category [HPS97, Section 4.4]). Given any $F$ that is small in the $K(n)$-local category (this means $[F,-]$ commutes with coproducts in the $K(n)$-local category), and any map $h: F \rightarrow X$, we define $U_{h}$ to be the set of all $f \in[X, Y]$ with $f h=0$. The $U_{f}$ define a basis of neighborhoods of 0 in $[X, Y]$, and then $V \subseteq[X, Y]$ is open if and only if for all $v \in V$, there is an $h$ such that $v+U_{h} \subseteq V$. Equivalently, $V$ is open if and only if for all $v$ in $V$ there is an $h$ such that $f h=v h$ implies $f \in V$.

This natural topology has a number of good properties. First of all, it is straightforward to check that the composition map

$$
[X, Y] \times[Y, Z] \rightarrow[X, Z]
$$

is continuous (see [HPS97, Proposition 4.4.1(a)]). The smash product map

$$
[X, Y] \times[Z, W] \rightarrow[X \widetilde{\wedge} Z, Y \widetilde{\wedge} W]
$$

is also continuous [HS99, Proposition 11.3], but it is slightly harder to check this. In good cases, the natural topology is the obvious one. For example, the natural topology on $E^{*} X$ and $E_{*}^{\vee} X$ is the $\mathfrak{m}$-adic topology when $K(n)_{*} X$ is finite-dimensional 
(that is, when $X$ is strongly dualizable in the $K(n)$-local category). This is proved in [HS99, Proposition 11.9] for a different version of $E$, but the proof is the same for our $E$.

There is a map

$$
\sigma: \Gamma \times E_{*}^{\vee} E \rightarrow E_{*}
$$

defined by letting $\sigma(\gamma, a)$ be the composite

$$
S^{m} \stackrel{a}{\rightarrow} E \widetilde{\wedge} E \stackrel{1 \widetilde{\wedge} \gamma}{\longrightarrow} E \widetilde{\wedge} E \stackrel{\mu}{\rightarrow} E .
$$

Now $E_{*}^{\vee} E, E_{*}$, and $\Gamma \subseteq[E, E]$ are sets of maps in the $K(n)$-local category, so they have a natural topology as described above. The map $\sigma$ is built from composition maps and the map

$$
[E, E] \rightarrow[E \widetilde{\wedge} E, E \widetilde{\wedge} E]
$$

that takes $\gamma$ to $1 \widetilde{\wedge} \gamma$. By the remarks above on the natural topology, we see that $\sigma$ is continuous. Its adjoint is therefore a map

$$
\Phi: E_{*}^{\vee} E \rightarrow C\left(\Gamma, E_{*}\right),
$$

where continuity is determined by the natural topologies. It is a straightforward diagram chase to verify that $\Phi$ is a map of $E_{*}$-modules. Using the fact that $\Gamma$ consists of ring spectrum automorphisms, one can also check by a diagram chase that $\Phi$ is a map of $E_{*}$-algebras.

We will of course need to determine the topological group $\Gamma$. For the moment, however, we content ourselves with some basic structural results.

Proposition 1.1. The set $[E, E]$ is a first countable compact Hausdorff topological ring in the natural topology, and the topology is defined by a set of open left ideals. Furthermore, the action of $[E, E]$ on $[X, E]$ is continuous for all $K(n)$-local spectra $X$.

Proof. The group $[X, Y]$ is always a topological group in the natural topology. Since the multiplication in $[E, E]$ is given by composition, it is continuous, and so $[E, E]$ is a topological ring. The same argument shows that the action of $[E, E]$ on $[X, E]$ is continuous. Now suppose $F$ is small in the $K(n)$-local category. The prototypical $F$ would be $L_{n} V(n-1)$, if it existed. This spectrum would have

$$
E^{*}\left(L_{n} V(n-1)\right) \cong E^{*} / \mathfrak{m},
$$

which is obviously finite in each degree. One of the main themes of [HS99] is that every small object in the $K(n)$-local category behaves as if it were built from finitely many copies of the putative spectrum $L_{n} V(n-1)$. In particular, the argument of [HS99, Theorem 8.6] shows that $E^{*} F$ is finite in each degree. Then $[\mathbf{H S 9 9}$, Proposition 11.5] implies that $[E, E]$ is profinite, and hence compact Hausdorff. To see that $[E, E]$ is first countable, note that there are only countably many (isomorphism classes of) small objects in the $K(n)$-local category by the results of [HS99, Section 8]. Given a small object $F$, we have just seen that $[F, E]$ is finite. Hence there are only countably many basic neighborhoods $U_{h}$ of 0 , so $[E, E]$ is first countable. It is clear that the $U_{h}$ are left ideals. 
Corollary 1.2. The group $\Gamma$ is a first countable Hausdorff topological group whose topology is defined by a set of open subgroups. Furthermore, the action of $\Gamma$ on $E_{*}$ is continuous when $E_{*}$ is given the $\mathfrak{m}$-adic topology.

Proof. In general, if $A$ is a topological ring defined by a set of open left ideals, then $A^{\times}$is a topological group defined by a set of open subgroups. Indeed, if $U$ is an open left ideal in $A$, then one can check that $V=(e+U) \cap A^{\times}$is an open subgroup in $A^{\times}$. If $\left\{U_{i}\right\}$ defines the topology on $A$, then the corresponding set $\left\{V_{i}\right\}$ defines the subspace topology on $A^{\times}$. It is clear that the multiplication map is continuous; to see that the inverse map is continuous, note that $\left(x\left(x^{-1} V x\right)\right)^{-1}=x^{-1} V$. Our group $\Gamma$ is a subgroup of $[E, E]^{\times}$, so is also a first countable Hausdorff topological group whose topology is defined by a set of open subgroups.

Proposition 1.1 shows that the action of $\Gamma$ on $E_{*}$ is continuous when $E_{*}$ is given the natural topology, and we have seen already that the natural topology on $E_{*}$ is the $\mathfrak{m}$-adic topology.

We want to conclude that $\Gamma$ is profinite. For this, we need to know that $\Gamma$ is compact, according to [DdSMS99, Definition 1.1]. Since $[E, E]$ is compact, it suffices to show that $\Gamma$ is closed in $[E, E]$.

Lemma 1.3. Suppose $A$ is a compact Hausdorff topological ring. Then $A^{\times}$is closed in $A$.

Proof. Note that $A^{\times}=B \cap C$, where $B$ is the set of all elements with a right inverse and $C$ is the set of all elements with a left inverse. We will show that $B$ is closed; a similar proof shows that $C$ is closed as well. Let $\mu: A \times A \rightarrow A$ denote the multiplication map, and let $D$ denote $\mu^{-1}(1)$, which is closed since $A$ is Hausdorff. Then $B=\pi_{1} D$, but $\pi_{1}$ is a map from the compact space $A \times A$ to the Hausdorff space $A$, so is a closed mapping.

Theorem 1.4. The group $\Gamma$ is a first countable profinite group.

Proof. In view of Corollary 1.2 and Lemma 1.3, it suffices to show that the set $S$ of ring spectrum maps in $[E, E]$ is closed. Note that $S=S_{1} \cap S_{2}$, where $S_{1}$ is the set of maps compatible with the multiplication $\mu$ and $S_{2}$ is the set of maps compatible with the unit $\eta$. Certainly $S_{2}$ is closed, since it is the inverse image of $\eta$ under the continuous composition

$$
[E, E] \rightarrow[S, E] \times[E, E] \stackrel{\circ}{\rightarrow}[S, E] .
$$

Here the first map sends $f$ to $(\eta, f)$, and the second map is composition. On the other hand, $S_{1}$ is the equalizer of the maps

$$
[E, E] \stackrel{\mu^{*}}{\longrightarrow}[E \wedge E, E]
$$

and

$$
[E, E] \stackrel{\Delta}{\longrightarrow}[E, E] \times[E, E] \stackrel{\wedge}{\longrightarrow}[E \wedge E, E \wedge E] \stackrel{\mu_{*}}{\longrightarrow}[E \wedge E, E] .
$$

The maps $\mu^{*}$ and $\mu_{*}$ are continuous since they are just composition, and the map $\wedge$ is continuous as well ([HS99, Proposition 11.3]). Proposition 11.5 of [HS99] tells 
us that $[E \wedge E, E]$ is Hausdorff, and the equalizer of two continuous maps into a Hausdorff space is always closed.

\section{Pro-free modules}

In this section, we show that both $E_{*}^{\vee} E$ and $C\left(\Gamma, E_{*}\right)$ are pro-free $E_{*}$-modules. This allows us to conclude that $\Phi$ is an isomorphism if and only if the induced map

$$
\Psi: K_{0} E \rightarrow C\left(\Gamma, \mathbb{F}_{p^{n}}\right)
$$

is an isomorphism. This section will use the results on pro-free modules from [HS99, Appendix A]. That appendix is completely algebraic and does not depend on the body of [HS99].

Recall that, if $R$ is a complete local ring, an $R$-module is called pro-free if it is the completion of a free module. Our particular ring $E_{*}$ is graded, so when we take the completion of a graded module we must do so in the graded sense. However, all our $E_{*}$-modules will be evenly graded, and since $E_{*}$ has a unit in degree 2 , there is an equivalence of categories between evenly graded $E_{*}$-modules and $E_{0}$-modules. This equivalence takes an evenly graded $E_{*}$-module $M$ to $M_{0}$, and an $E_{0}$-module $N$ to $N\left[u, u^{-1}\right]$, with $N$ in degree 0 .

We need to show that the $E_{*}$-modules we deal with are pro-free. For this, we recall the spectrum $K=E / \mathfrak{m}$. This spectrum can be made into a ring spectrum by using, for example, the theory of bordism with singularities or its modern replacement [EKMM97, Chapter V]. It is in fact a field spectrum, and is additively isomorphic to a wedge of suspensions of $K(n)$. Note that $K_{*} \cong \mathbb{F}_{p^{n}}\left[u, u^{-1}\right]$.

The following lemma is very useful.

Lemma 2.1. If $K_{*} X$ is concentrated in even degrees, then $E^{*} X$ and $E_{*}^{\vee} X$ are profree $E_{*}$-modules. Furthermore, in this case $K_{*} X \cong\left(E_{*}^{\vee} X\right) / \mathfrak{m}$ and $K^{*} X \cong E^{*} X / \mathfrak{m}$.

Proof. This lemma is proven, for a slightly different spectrum $E$, in Propositions 2.5 and $8.4(\mathrm{f})$ of [HS99]. Let $G(k)$ denote the theory $E /\left(p, u_{1}, \ldots, u_{k-1}\right)$. There is an obvious exact sequence relating $G(k)$ to $G(k+1)$. Using these exact sequences, one can work back from $K=G(n)$ to $E=G(0)$. In so doing, one sees that $G(k)_{*}^{\vee} X$ is evenly graded and that $G(k+1)_{*}^{\vee}(X) \cong G(k)_{*}^{\vee}(X) / u_{k}$. Hence $K_{*} X \cong\left(E_{*}^{\vee} X\right) / \mathfrak{m}$. It also follows that $\left(p, u_{1}, \ldots, u_{n-1}\right)$ is a regular sequence on $E_{*}^{\vee} X$. Theorem A.9 of [HS99] then guarantees that $E_{*}^{\vee} X$ is pro-free. The same method works for $E^{*} X$ as well.

Proposition 2.2. The $E_{*}$-module $E_{*}^{\vee} E$ is pro-free and concentrated in even dimensions.

Proof. By Lemma 2.1, it suffices to check that $K_{*} E$ is concentrated in even degrees. Landweber exactness of $E$ implies that $K_{*} E \cong K_{*} B P \otimes_{B P_{*}} E_{*}$, but the AtiyahHirzebruch spectral sequence implies that $K_{*} B P \cong K_{*}\left[t_{1}, t_{2}, \ldots\right]$ with $\left|t_{i}\right|=2\left(p^{i}-\right.$ 1) as in [Rav86, Lemma 4.1.7].

To show that $C\left(\Gamma, E_{*}\right)$ is pro-free will require more work. We begin by analyzing $C(G, R)$ when $G$ is profinite and $R$ is discrete. 
Lemma 2.3. Suppose $G=\lim G / U$ is a profinite group and $R$ is a discrete commutative ring. Then the natural map of rings $\rho: \operatorname{colim}_{U} F(G / U, R) \rightarrow C(G, R)$ is an isomorphism, where $U$ runs through the open normal subgroups of $G$,

This lemma works when $R$ is graded as well.

Proof. The map $\rho$ sends a map $G / U \rightarrow R$ to the composite $G \rightarrow G / U \rightarrow R$. Since the reduction maps are surjective, $\rho$ is injective. Now suppose $f: G \rightarrow R$ is continuous. For each $x \in G$, choose an open normal subgroup $N_{x}$ such that $f\left(x N_{x}\right)=f(x)$. The $x N_{x}$ form an open cover of $G$, so there is a finite subcover $\left\{x_{1} N_{x_{1}}, \ldots, x_{k} N_{x_{k}}\right\}$. Let $N=N_{x_{1}} \cap \cdots \cap N_{x_{k}}$. We claim that $f$ is induced by a function $G / N \rightarrow \mathbb{Z}$. Indeed, suppose $x$ and $y$ are congruent modulo $N$. Now $x$ must lie in some $x_{i} N_{x_{i}}$, so $x$ is congruent to $x_{i}$ modulo $N_{x_{i}}$. It follows that $y$ is also congruent to $x_{i}$ modulo $N_{x_{i}}$, and so $f(x)=f\left(x_{i}\right)=f(y)$, as required.

In fact, the dependence of $C(G, R)$ on $R$ is very simple.

Proposition 2.4. Suppose $G$ is a profinite group and $R$ is a discrete commutative ring. Then the natural map $\sigma: R \otimes C(G, \mathbb{Z}) \rightarrow C(G, R)$ defined by $\sigma(r, f)(g)=f(g) r$ is an isomorphism.

Again, this proposition holds if $R$ is graded as well, where $C(G, \mathbb{Z})$ is thought of as a graded ring concentrated in degree 0 .

Proof. When $G$ is finite, the proposition is clear since both sides are free $R$-modules of rank $|G|$, and $\sigma$ takes the basis $\left\{1 \otimes g^{*}\right\}$ to the basis $\left\{g^{*}\right\}$, where $g^{*}$ is the function that takes $g$ to 1 and everything else to 0 . When $G=\lim (G / U)$ is profinite, we take the direct limits of the isomorphisms for $G / U$ and use Lemma 2.3.

We need a version of Proposition 2.4 when $R$ is complete in the a-adic topology, for some ideal a. In this case, if $S$ is a discrete ring such as $C(G, \mathbb{Z})$, we define $R \widehat{\otimes} S$ to be the completion of $R \otimes S$ with respect to the image of $\mathfrak{a} \otimes S$ in $R \otimes S$.

Proposition 2.5. Suppose $G$ is a profinite group and $R$ is a commutative ring that is complete in the $\mathfrak{a}$-adic topology for some ideal $\mathfrak{a}$. Then there is a natural isomorphism

$$
R \widehat{\otimes} C(G, \mathbb{Z}) \stackrel{\cong}{\rightrightarrows} C(G, R) .
$$

As usual, this isomorphism will work in the graded case as well, as long as $\mathfrak{a}$ is homogeneous.

Proof. Take the inverse limit of the isomorphisms

$$
\sigma: R / \mathfrak{a}^{i} \otimes C(G, \mathbb{Z}) \rightarrow C\left(G, R / \mathfrak{a}^{i}\right),
$$

of Proposition 2.4.

We can now prove that $C\left(\Gamma, E_{*}\right)$ is pro-free.

Theorem 2.6. Suppose $G$ is a first countable profinite group and $R$ is a commutative ring that is complete in the $\mathfrak{a}$-adic topology for some ideal $\mathfrak{a}$. Then $C(G, R)$ is pro-free as an $R$-module. 
This theorem holds in either the graded or ungraded case.

Proof. Proposition 2.5 tells us that $C(G, R) \cong R \widehat{\otimes} C(G, \mathbb{Z})$. Hence it suffices to show that $C(G, \mathbb{Z})$ is a free abelian group. But Lemma 2.3 tells us that

$$
C(G, \mathbb{Z}) \cong \operatorname{colim}_{U} F(G / U, \mathbb{Z}) .
$$

Since $G$ is first countable, we can make this colimit run over a chain

$$
\cdots \subseteq U_{k} \subseteq \cdots \subseteq U_{0}
$$

Each group $F\left(G / U_{k}, \mathbb{Z}\right)$ is a finitely generated free abelian group, and each map $F\left(G / U_{k}, \mathbb{Z}\right) \rightarrow F\left(G / U_{k+1}, \mathbb{Z}\right)$ is a split monomorphism. Hence $C(G, \mathbb{Z})$ is a free abelian group.

We then have the following general lemma about maps of pro-free modules.

Lemma 2.7. Suppose $R$ is a Noetherian regular complete local ring with maximal ideal $\mathfrak{m}$. A map $f: M \rightarrow N$ of pro-free modules is an isomorphism if and only $R / \mathfrak{m} \otimes_{R} f$ is an isomorphism.

We are of course interested in this lemma when $R$ is graded, but the proof is the same in any case.

Proof. It is proved in [HS99, Proposition A.13] that if $R / \mathfrak{m} \otimes_{R} f$ is a monomorphism, then $f$ is a split monomorphism. The cokernel $C$ of $f$ is then a summand in the complete module $N$, but $C / \mathfrak{m} C=0$. It follows that $C=0$.

We then have the following theorem.

Theorem 2.8. The map $\Phi: E_{*}^{\vee} E \rightarrow C\left(\Gamma, E_{*}\right)$ is an isomorphism if and only if the map $\Psi: K_{0} E \rightarrow C\left(\Gamma, \mathbb{F}_{p^{n}}\right)$ induced by $\Phi$ is an isomorphism.

Proof. Since $E_{*}^{\vee} E$ and $C\left(\Gamma, E_{*}\right)$ are pro-free by Proposition 2.2 and Theorem 2.6, Lemma 2.7 implies that $\Phi$ is an isomorphism if and only $\Phi / \mathfrak{m}$ is an isomorphism. Lemma 2.1 then tells us that $E_{*}^{\vee} E / \mathfrak{m} \cong K_{*} E$. Proposition 2.5 and Proposition 2.4 imply that

$$
\begin{gathered}
C\left(\Gamma, E_{*}\right) / \mathfrak{m} \cong\left(E_{*} \widehat{\otimes} C(\Gamma, \mathbb{Z})\right) / \mathfrak{m} \\
\cong\left(E_{*} \otimes C(\Gamma, \mathbb{Z})\right) / \mathfrak{m} \cong\left(E_{*} / \mathfrak{m}\right) \otimes C(\Gamma, \mathbb{Z}) \cong C\left(\Gamma, K_{*}\right) .
\end{gathered}
$$

Now $K_{*} E$ is concentrated in even dimensions by Proposition 2.2 , as is $C\left(\Gamma, K_{*}\right)$. Since both contain the unit $u$ in degree $2, \Phi / \mathfrak{m}$ is an isomorphism if and only if the degree 0 part $\Psi$ of $\Phi / \mathfrak{m}$ is an isomorphism.

\section{Ind-étale algebras}

The object of this section is to show that

$$
\Psi: K_{0} E \rightarrow C\left(\Gamma, \mathbb{F}_{p^{n}}\right)
$$

is an isomorphism if and only if $\mathbf{A l g}_{\mathbb{F}_{p^{n}}}\left(\Psi, \overline{\mathbb{F}_{p}}\right)$ is an isomorphism of sets, where $\overline{\mathbb{F}_{p}}$ is the algebraic closure of $\mathbb{F}_{p}$, and $\mathbf{A l g}_{\mathbb{F}_{p^{n}}}$ is the category of $\mathbb{F}_{p^{n} \text {-algebras. We will }}$ 


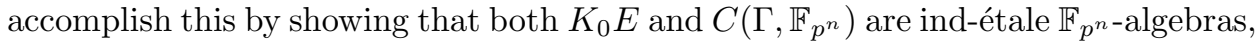
and proving a general result about maps between such algebras.

\subsection{Ind-étale algebras}

We first develop the algebraic theory of ind-étale algebras. For a general commutative ring $k$, a finitely presented $k$-algebra is called étale if, whenever $N$ is a square 0 ideal in a $k$-algebra $C$, the natural map

$$
\operatorname{Alg}_{k}(A, C) \rightarrow \mathbf{A l g}_{k}(A, C / N)
$$

is an isomorphism. $A$ is called smooth if this map is always surjective, and unramified if it is always injective. These definitions are taken from [DG70, Section I.4.3], where of course one works with arbitrary schemes.

If $k$ is a field, the category of étale $k$-schemes is equivalent to the category of $G$ sets, where $G$ is the Galois group of the separable closure $k_{s}$ over $k$ [DG70, I.4.6.4]. Note that $G$ is profinite, and so a $G$-set means a set with a continuous action of $G$. The equivalence takes $X$ to the set $X\left(k_{s}\right)$ of its $k_{s}$-valued points. The equivalence works because if $X$ is an étale $k$-scheme, then $X \otimes_{k} k_{s}$ is constant [DG70, I.4.6.2]. Now, if $A$ is an étale algebra, then $\operatorname{Spec} A \otimes_{k} k_{s}$ is both constant and affine, so must be a finite constant scheme. Thus we have the following well-known proposition. See Proposition 18.3 and Theorem 18.4 of [KMRT98].

Proposition 3.1. Suppose $k$ is a field. $A$ k-algebra $A$ is étale if and only if $A \otimes_{k} k_{s}$ is isomorphic to a finite product of copies of $k_{s}$. Moreover, the category of étale $k$-algebras is anti-equivalent to the category of finite $G$-sets, where $G$ denotes the Galois group of $k_{s} / k$.

Now, the algebras we have to deal with are definitely not finitely presented, so we make the following definition.

Definition 3.2. If $k$ is a commutative ring, we define a $k$-algebra $A$ to be ind-étale if $A$ is a filtered colimit of étale $k$-algebras.

The main reason we introduce this definition is the following lemma.

Lemma 3.3. If $k$ is a field and $G$ is a profinite group, then $C(G, k)$ is an ind-étale $k$-algebra.

Proof. Lemma 2.3 tells us that $C(G, k) \cong \operatorname{colim} F(G / U, k)$, where $U$ runs through the open normal subgroups of $G$. Each $F(G / U, k)$ is obviously étale, since it is a product of copies of $k$ even before tensoring with $k_{s}$.

The terminology "ind-étale" is justified by the following proposition.

Proposition 3.4. For a commutative ring $k$, the category of ind-étale $k$-algebras is equivalent to the category of ind-objects in the category of étale k-algebras.

Here, given a category $\mathcal{C}$, the category of ind-objects in $\mathcal{C}$ is the category of all functors $F: I \rightarrow \mathcal{C}$, where $I$ can be any filtered small category. The morphisms in this category from $F$ to $G$ are defined to be $\lim _{\alpha} \operatorname{colim}_{\beta} \mathcal{C}(F(\alpha), G(\beta))$. See [SGA4, Section I.8.2]. 
Proof. The colimit is an obvious functor from ind-objects to ind-étale $k$-algebras. It is essentially surjective by definition. To see that it is fully faithful, we compute

$$
\begin{gathered}
\operatorname{Alg}_{k}\left(\operatorname{colim} A_{\alpha}, \operatorname{colim} B_{\beta}\right) \cong \lim _{\alpha} \operatorname{Alg}_{k}\left(A_{\alpha}, \operatorname{colim} B_{\beta}\right) \\
\cong \lim _{\alpha} \operatorname{colim}_{\beta} \operatorname{Alg}_{k}\left(A_{\alpha}, B_{\beta}\right),
\end{gathered}
$$

using the fact that $A_{\alpha}$ is finitely presented as a $k$-algebra.

It follows from Proposition 3.1 that, if $k$ is a field, the category of ind-étale $k$ algebras is anti-equivalent to the category of pro-objects in the category of finite $G$-sets.

Proposition 3.5. Let $G$ be a profinite group. The limit functor from the category of pro-objects in finite $G$-sets to profinite $G$-sets is an equivalence of categories.

The morphisms in the category of profinite $G$-sets are continuous equivariant maps from $X$ to $Y$, denoted $C_{G}(X, Y)$.

Proof. The limit functor is essentially surjective by definition. To determine whether it is fully faithful, we compute

$$
C_{G}\left(\lim X_{\alpha}, \lim Y_{\beta}\right) \cong \lim _{\beta} C_{G}\left(\lim X_{\alpha}, Y_{\beta}\right) .
$$

There is a canonical map

$$
\sigma: \operatorname{colim}_{\alpha} C_{G}\left(X_{\alpha}, Y_{\beta}\right) \rightarrow C_{G}\left(\lim X_{\alpha}, Y_{\beta}\right) .
$$

We claim that this map is an isomorphism. To see that $\sigma$ is surjective, note that a continuous $G$-map $f: \lim X_{\alpha} \rightarrow Y_{\beta}$ is determined by a partition of $\lim X_{\alpha}$ into open and closed sets $U_{y}=f^{-1}(y)$ for $y \in Y_{\beta}$, with $g U_{y} \subseteq U_{g y}$. A basis for the topology on $\lim X_{\alpha}$ is given by the sets $\pi_{\gamma}^{-1}\left(V_{\gamma}\right)$, where $\pi_{\gamma}: \lim X_{\alpha} \rightarrow X_{\gamma}$ is the evident map and $V_{\gamma}$ is an arbitrary subset of $X_{\gamma}$. Because our indexing category is filtered, this basis is closed under finite intersections and finite unions. Since $\lim X_{\alpha}$ is compact, this means that a set that is both open and closed must be of the form $\pi_{\gamma}^{-1}\left(V_{\gamma}\right)$. Again using the fact that the indexing category is filtered, we conclude that there is a $\gamma$ and subsets $V_{y}$ in $X_{\gamma}$ such that $U_{y}=\pi_{\gamma}^{-1}\left(V_{y}\right)$ for all $y \in Y_{\beta}$. It follows that $f$ is in the image of $C_{G}\left(X_{\gamma}, Y_{\beta}\right)$, and hence that $\sigma$ is surjective. This implies that the inverse limit functor is full.

To see that $\sigma$ is injective, we use an argument we learned from Neil Strickland. Suppose we have two maps $f: X_{\alpha} \rightarrow Y_{\beta}$ and $f^{\prime}: X_{\alpha^{\prime}} \rightarrow Y_{\beta}$ with $f \circ \pi_{\alpha}=f^{\prime} \circ \pi_{\alpha^{\prime}}$. Since the diagram $\left\{X_{\gamma}\right\}$ is filtered, we can assume $\alpha=\alpha^{\prime}$. Choose an $x \in X_{\alpha}$ with $f(x) \neq f^{\prime}(x)$. Let $\mathcal{I}$ denote the indexing category of the pro-object $X$, and let $\mathcal{C}$ denote the category of all pairs $(\gamma, i)$ where $i: \gamma \rightarrow \alpha$ is a morphism in $\mathcal{I}$. Then $\mathcal{C}$ is itself a filtered category, and we can define a functor from $\mathcal{C}$ to finite sets by taking $(\gamma, i)$ to $(X(i))^{-1}(x)$. The inverse limit of this functor is $\pi_{\alpha}^{-1}(x)$, which must be empty since $f \circ \pi_{\alpha}=f^{\prime} \circ \pi_{\alpha}$. By [DdSMS99, Proposition 1.1.4], it follows that there exists an $i: \gamma \rightarrow \alpha$ such that $(X(i))^{-1}(x)=\emptyset$. Since there are only finitely many elements $x$ and $\mathcal{I}$ is filtered, it follows that there exists a morphism $j: \delta \rightarrow \alpha$ such that $(X(j))^{-1}(x)$ is empty for all $x$ with $f(x) \neq f^{\prime}(x)$. Hence $f \circ X(j)=f^{\prime} \circ X(j)$, and so $f$ and $f^{\prime}$ represent the same element of $\operatorname{colim}_{\alpha} C_{G}\left(X_{\alpha}, Y_{\beta}\right)$. 
We reach the following conclusion.

Theorem 3.6. Suppose $k$ is a field with separable closure $k_{s}$. Let $G$ be the Galois group of $k_{s}$ over $k$. The functor that takes a $k$-algebra $A$ to $\mathbf{A l g}_{k}\left(A, k_{s}\right)$ defines an anti-equivalence of categories between ind-étale $k$-algebras and profinite $G$-sets. In particular, if $f: A \rightarrow B$ is a map of ind-étale $k$-algebras, then $f$ is an isomorphism if and only if the induced map

$$
\operatorname{Alg}_{k}\left(B, k_{s}\right) \rightarrow \mathbf{A l g}_{k}\left(A, k_{s}\right)
$$

is an isomorphism.

\subsection{Identification of $K_{0} E$}

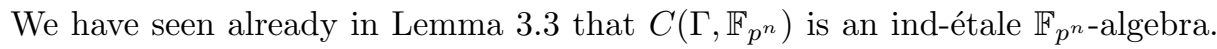

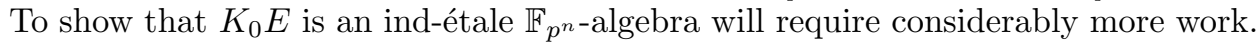
The goal of this section is to show that

$$
K_{0} E \cong \mathbb{F}_{p^{n}} \otimes_{V} V T\left[t_{0}, t_{0}^{-1}\right] \otimes_{V} \mathbb{F}_{p^{n}}
$$

where $V$ is $B P_{*}$ thought of as an ungraded ring, and $V T$ is $B P_{*} B P$ thought of as an ungraded ring. Thus $\left(V, V T\left[t_{0}, t_{0}^{-1}\right]\right)$ is the Hopf algebroid that represents the groupoid of $p$-typical formal group laws and arbitrary isomorphisms between them (see [Rav86, p. 365]).

We begin by defining $R_{*}=B P_{*}\left[u, u^{-1}\right]$, where $u$ has degree 2 . Then $R_{*}$ is a free $B P_{*}$-algebra, so is obviously Landweber exact. Hence we get a commutative ring spectrum $R$, and an isomorphism $R_{*} X \cong R_{*} \otimes_{B P_{*}} B P_{*} X$. This is easy to see, but see [HS99, Section 2] for details on Landweber exact spectra. In particular, $R_{*} R \cong R_{*} \otimes_{B P_{*}} B P_{*} B P \otimes_{B P_{*}} R_{*}$.

Lemma 3.7. There is an isomorphism of Hopf algebroids

$$
\tau:\left(V\left[u, u^{-1}\right], V T\left[t_{0}, t_{0}^{-1}\right]\left[u, u^{-1}\right]\right) \cong\left(R_{*}, R_{*} R\right) .
$$

Proof. A map of graded rings from $R_{*}$ to $S$ is a pair $(F(x, y), u)$ where $F(x, y)$ is a homogeneous $p$-typical formal group law over $S$ and $u$ is a unit in $S_{2}$. To say that $F(x, y)=\sum_{i, j} a_{i j} x^{i} y^{j}$ is homogeneous means that each $a_{i j}$ is homogeneous of degree $2(i+j-1)$. On the other hand, a map of graded rings from $V\left[u, u^{-1}\right]$ to $S$ is a pair $\left(F_{0}(x, y), y\right)$ where $F_{0}$ is $p$-typical formal group law over $S_{0}$ and $u$ is a unit in $S_{2}$. Given $(F(x, y), u)$ we define

$$
F_{0}(x, y)=\sum_{i, j} a_{i j} u^{1-i-j} x^{i} y^{j}=u F\left(u^{-1} x, u^{-1} y\right) .
$$

One can readily see from [Rav86, Lemma A2.1.26] that $F_{0}$ is $p$-typical. Conversely, given $\left(F_{0}(x, y), u\right)$ we define $F(x, y)=u^{-1} F_{0}(u x, u y)$, which is homogeneous and $p$-typical. This one-to-one correspondence gives us the desired isomorphism $V\left[u, u^{-1}\right] \cong R_{*}$.

Similarly, a map of graded rings from $R_{*} R$ to $S$ is a quintuple

$$
\left(F(x, y), F^{\prime}(x, y), u, v, \phi\right) \text {, }
$$


where $F(x, y)$ and $F^{\prime}(x, y)$ are homogeneous $p$-typical formal group laws over $S$, $u, v$ are units in $S_{2}$, and $\phi$ is a homogeneous strict isomorphism from $F$ to $F^{\prime}$, in the sense that $\phi(x)=\sum b_{i} x^{i}$ with $b_{i}$ homogeneous of degree $2 i-2$. On the other hand, a map of graded rings from $V T\left[t_{0}, t_{0}^{-1}\right]\left[u, u^{-1}\right]$ to $S$ is a quadruple $\left(F_{0}(x, y), F_{0}^{\prime}(x, y), u, \psi\right)$, where $F_{0}$ and $F_{0}^{\prime}$ are formal group laws over $S_{0}, u$ is a unit in degree 2 , and $\psi$ is an arbitrary isomorphism from $F_{0}$ to $F_{0}^{\prime}$. Given $\left(F, F^{\prime}, u, v, \phi\right)$, we define $F_{0}(x, y)=u F\left(u^{-1} x, u^{-1} y\right)$ and $F_{0}^{\prime}(x, y)=v F^{\prime}\left(v^{-1} x, v^{-1} y\right)$ as above. We define $\psi(x)=v \phi\left(u^{-1} x\right)$. The reader can check that $\psi$ is defined over $S_{0}$, and has leading term $v u^{-1} x$. We have

$$
\begin{gathered}
F_{0}^{\prime}(\psi x, \psi y)=v F^{\prime}\left(v^{-1} \psi x, v^{-1} \psi y\right)=v F^{\prime}\left(\phi\left(u^{-1} x\right), \phi\left(u^{-1} y\right)\right) \\
=v \phi F\left(u^{-1} x, u^{-1} y\right)=v \phi\left(u^{-1} F_{0}(x, y)\right)=\psi F_{0}(x, y) .
\end{gathered}
$$

Hence $\psi$ is an isomorphism from $F_{0}$ to $F_{0}^{\prime}$. Conversely, given $\left(F_{0}, F_{0}^{\prime}, u, \psi\right)$. we let $w$ be the leading coefficient $(d \psi(x) / d x)(0)$ of $\psi$ and define

$$
v=u w, F(x, y)=u^{-1} F(u x, u y), F^{\prime}=v^{-1} F_{0}^{\prime}(v x, v y) \text {, and } \phi(x)=v^{-1} \psi(u x) .
$$

We leave to the reader the check that $\phi(x)$ is a homogeneous strict isomorphism, and that these constructions are inverse to one another.

We then have the following proposition.

Proposition 3.8. $E_{0} E \cong E_{0} \otimes_{V} V T\left[t_{0}, t_{0}^{-1}\right] \otimes_{V} E_{0}$.

Note that $E_{0}$ is a $V$-algebra via the map that takes $v_{i}$ to $u_{i}$ for $0 \leqslant i<n$, takes $v_{n}$ to 1 , and takes $v_{i}$ to 0 for $i>n$.

Proof. The map $B P_{*} \rightarrow E_{*}$ used to build $E_{*}(-)$ factors through $R_{*}$. Using Landweber exactness of $E$, we conclude that

$$
E_{*} E \cong E_{*} \otimes_{R_{*}} R_{*} R \otimes_{R_{*}} E_{*} .
$$

Using Lemma 3.7, we conclude that

$$
\begin{aligned}
& E_{0} E\left[u, u^{-1}\right] \cong E_{*} E \cong E_{0}\left[u, u^{-1}\right] \otimes_{V\left[u, u^{-1}\right]} V T\left[t_{0}, t_{0}^{-1}\right]\left[u, u^{-1}\right] \otimes_{V\left[u, u^{-1}\right]} E_{0}\left[u, u^{-1}\right] \\
& \cong\left(E_{0} \otimes_{V} V T\left[t_{0}, t_{0}^{-1}\right] \otimes_{V} E_{0}\right)\left[u, u^{-1}\right] .
\end{aligned}
$$

The proposition follows.

Corollary 3.9. $K_{0} E \cong \mathbb{F}_{p^{n}} \otimes_{V} V T\left[t_{0}, t_{0}^{-1}\right] \otimes_{V} \mathbb{F}_{p^{n}}$.

In this corollary, the map $V \rightarrow \mathbb{F}_{p^{n}}$ sends all $v_{i}$ to 0 except $v_{n}$, which goes to 1 .

Proof. Because $E$ is Landweber exact, $E_{*} E$ is a flat $E_{*}$-module. In particular, by tensoring the sequences

$$
0 \rightarrow E_{*} /\left(p, u_{1}, \ldots, u_{i-1}\right) \stackrel{u_{i}}{\longrightarrow} E_{*} /\left(p, u_{1}, \ldots, u_{i-1}\right) \rightarrow E_{*} /\left(p, u_{1}, \ldots, u_{i}\right) \rightarrow 0
$$

with $E_{*} E$, we see that $\left(p, u_{1}, \ldots, u_{n-1}\right)$ is a regular sequence on $E_{*} E$. Hence $K_{*} E \cong$ $E_{*} E / \mathfrak{m}$, and so $K_{0} E \cong E_{0} E / \mathfrak{m}$. Since $I_{n}=\left(p, v_{1}, \ldots, v_{n-1}\right)$ is an invariant ideal in $V T$, the corollary follows from Proposition 3.8. 


\section{3. $\quad K_{0} E$ as an ind-étale $\mathbb{F}_{p^{n}}$-algebra}

Having identified $K_{0} E$, we can begin the process of proving that $K_{0} E$ is an ind-étale $\mathbb{F}_{p^{n}}$-algebra. We begin by identifying the representing scheme for automorphisms of the Honda formal group law $F_{n}$. Recall that this is the $p$-typical formal group law over an $\mathbb{F}_{p}$-algebra $S$ whose classifying map takes $v_{i}$ to 0 for all $i \neq n$ and takes $v_{n}$ to 1 . Its $p$-series is $[p]_{F_{n}}(x)=x^{p^{n}}$.

Proposition 3.10. Let $S$ be an $\mathbb{F}_{p}$-algebra. A power series $\psi(x) \in S[[x]]$ is an automorphism of the Honda formal group law $F_{n}$ if and only if $\psi^{-1}(x)=\sum_{i=0}^{\infty} F_{n} t_{i} x^{p^{i}}$ where $t_{i}^{p^{n}}=t_{i}$ for all $i$ and $t_{0}$ is a unit.

The only if half of this proposition is proved in the proof of [Str00, Theorem 12], and the if half is stated there.

Proof. Suppose first that $\psi(x)$ is an automorphism of $F_{n}$. Since $F_{n}$ is $p$-typical, Lemma A2.1.26 of $[\mathbf{R a v 8 6}]$ implies that $\psi^{-1}(x)=\sum_{i=0}^{\infty} F_{n} t_{i} x^{p^{i}}$. Since $\psi^{-1}(x)$ is also an automorphism of $F_{n}$, it commutes with the $p$-series. We conclude that

$$
\sum_{i=0}^{\infty} F_{n} t_{i}\left(x^{p^{n}}\right)^{p^{i}}=\left(\psi^{-1}(x)\right)^{p^{n}}
$$

so

$$
\sum_{i=0}^{\infty} F_{n} t_{i} x^{p^{i+n}}=\sum_{i=0}^{\infty} F_{n} t_{i}^{p^{n}} x^{p^{i+n}}
$$

Hence $t_{i}^{p^{n}}=t_{i}$ for all $i$.

Conversely, suppose $\psi^{-1}(x)=\sum_{i=0}^{\infty} F_{n} t_{i} x^{p^{i}}, t_{i}^{p^{n}}=t_{i}$ for all $i$, and $t_{0}$ is a unit. Define $F(x, y)=\psi F_{n}\left(\psi^{-1} x, \psi^{-1} y\right)$. Then $F$ is a formal group law, and $\psi$ is an isomorphism from $F_{n}$ to $F$. Furthermore, $F$ is $p$-typical by [Rav86, Lemma A2.1.26]. Thus $[p]_{F}(x)=\sum_{j=1}^{\infty} F s_{j} x^{p^{j}}$ for some elements $s_{j}$ of $S$. Note that $s_{j}$ is the image of $v_{j}$ under the classifying map of $F$, so it suffices to show that $s_{j}=0$ for all $j$ except $n$, and that $s_{n}=1$.

We do this by comparing the $p$-series. On the one hand, we have

$$
\psi^{-1}[p]_{F}(x)=\psi^{-1}\left(\sum_{j=1}^{\infty} s_{j} x^{p^{j}}\right)=\sum_{j=1}^{\infty} F_{n} \psi^{-1}\left(s_{j} x^{p^{j}}\right)=\sum_{i=0}^{\infty} F_{n} \sum_{j=1}^{\infty} F_{n} t_{i} s_{j}^{p^{i}} x^{p^{i+j}} .
$$

On the other hand, we have

$$
\psi^{-1}[p]_{F}(x)=[p]_{F_{n}}\left(\psi^{-1} x\right)=\sum_{i=0}^{\infty} F_{n} t_{i} x^{p^{i+n}}
$$


using the fact that $t_{i}^{p^{n}}=t_{i}$. Looking at the smallest power of $x$ that occurs, we conclude that $t_{0} s_{1}=0$ (if $n>1$ ), so $s_{1}=0$ since $t_{0}$ is a unit. Continuing in this fashion we see that $s_{j}=0$ for $j<n$ and $s_{n}=1$. Hence

$$
\sum_{i=0}^{\infty} F_{n} t_{i} x^{p^{i+n}}+{ }_{F_{n}} \sum_{i=0}^{\infty} F_{n} \sum_{j=n+1}^{\infty} F_{n} t_{j} s_{j}^{p^{i}} x^{p^{i+j}}=\sum_{i=0}^{\infty} F_{n} t_{i} x^{p^{i+n}} .
$$

Cancelling and again recursively looking at the smallest power of $x$ that occurs, we find that $s_{j}=0$ for all $j>n$ as well.

Corollary 3.11. The representing ring for automorphisms of the Honda formal group law $F_{n}$ is $\mathbb{F}_{p}\left[t_{0}, t_{1}, \ldots\right] /\left(t_{0}^{p^{n}-1}-1, t_{1}^{p^{n}}-t_{1}, \ldots\right)$.

We then get the following description of $K_{0} E$, also obtained by Strickland in the proof of [Str00, Theorem 12].

Proposition 3.12. $K_{0} E \cong \mathbb{F}_{p^{n}}\left[t_{0}, t_{1}, \ldots\right] /\left(t_{0}^{p^{n}-1}-1, t_{1}^{p^{n}}-t_{1}, \ldots\right) \otimes \mathbb{F}_{p^{n}}$.

Proof. By Corollary 3.9, we have

$$
K_{0} E \cong \mathbb{F}_{p^{n}} \otimes_{V} V T\left[t_{0}, t_{0}^{-1}\right] \otimes_{V} \mathbb{F}_{p^{n}}
$$

where the map $V \rightarrow \mathbb{F}_{p^{n}}$ takes $u_{i}$ to 0 for all $i \neq n$ and takes $u_{n}$ to 1 . This ring represents the functor that assigns to a ring $R$ the set of triples $(r, s, \psi)$, where $r, s: \mathbb{F}_{p^{n}} \rightarrow R$ are ring homomorphisms and $\psi$ is an isomorphism of formal groups from $r_{*} F_{n}$ to $s_{*} F_{n}$, where $F_{n}$ is the Honda formal group law classified by the map $V \rightarrow \mathbb{F}_{p^{n}}$. In fact, $F_{n}$ is actually defined over $\mathbb{F}_{p}$, so $r_{*} F_{n}=s_{*} F_{n}=F_{n}$. We conclude from Corollary 3.11 that

$$
K_{0} E \cong \mathbb{F}_{p^{n}} \otimes \mathbb{F}_{p}\left[t_{0}, t_{1}, \ldots\right] /\left(t_{0}^{p^{n}-1}-1, t_{1}^{p^{n}}-t_{1}, \ldots\right) \otimes \mathbb{F}_{p^{n}},
$$

which proves the proposition.

Theorem 3.13. $K_{0} E$ is an ind-étale $\mathbb{F}_{p^{n} \text {-algebra. }}$

Proof. Define

$$
R_{k}^{\prime}=\mathbb{F}_{p^{n}}\left[t_{0}, t_{1}, \ldots, t_{k}\right] /\left(t_{0}^{p^{n}-1}-1, t_{1}^{p^{n}}-t_{1}, \ldots, t_{k}^{p^{n}}-t_{k}\right)
$$

and define $R_{k}=R_{k}^{\prime} \otimes \mathbb{F}_{p^{n}}=R_{k}^{\prime} \otimes_{\mathbb{F}_{p^{n}}}\left(\mathbb{F}_{p^{n}} \otimes \mathbb{F}_{p^{n}}\right)$. It is clear from Proposition 3.12 that $K_{0} E \cong \operatorname{colim} R_{k}$ as $\mathbb{F}_{p^{n}}$-algebras. Thus it suffices to show that $R_{k}$ is an étale $\mathbb{F}_{p^{n} \text {-algebra. Since }}$

$$
\operatorname{Alg}_{\mathbb{F}_{p^{n}}}\left(R_{k},-\right) \cong \operatorname{Alg}_{\mathbb{F}_{p^{n}}}\left(R_{k}^{\prime},-\right) \times \operatorname{Alg}_{\mathbb{F}_{p^{n}}}\left(\mathbb{F}_{p^{n}} \otimes \mathbb{F}_{p^{n}},-\right),
$$

it suffices to show that both $R_{k}^{\prime}$ and $\mathbb{F}_{p^{n}} \otimes \mathbb{F}_{p^{n}}$ are étale. It is easy to check that

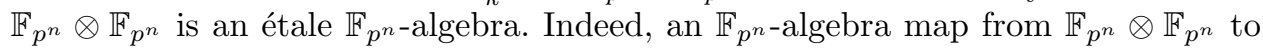
$A$ is the same thing as a ring map $\mathbb{F}_{p^{n}} \rightarrow A$. Now suppose $N$ is a square-zero ideal in $A$. If $f, g: \mathbb{F}_{p^{n}} \rightarrow A$ become equal in $A / N$, then $(f(x)-g(x))^{p^{n}}=0$, and so $f\left(x^{p^{n}}\right)=g\left(x^{p^{n}}\right)$ for all $x \in \mathbb{F}_{p^{n}}$. Since $x^{p^{n}}=x$, we conclude that $f=g$. Conversely, if $\bar{f}: \mathbb{F}_{p^{n}} \rightarrow A / N$ is a ring map, we define $f(x)$ for $x \in \mathbb{F}_{p^{n}}$ as follows. Write $\bar{f}(x)=z+N$ for some $z \in A$, and define $f(x)=z^{p^{n}}$, One can readily verify that this is independent of the choice of $z$, and is therefore a ring map that lifts $\bar{f}$. 
The proof that $R_{k}^{\prime}$ is étale is very similar. If $f, g: R_{k}^{\prime} \rightarrow A$ become equal in $A / N$, then again $f\left(x^{p^{n}}\right)=g\left(x^{p^{n}}\right)$ for all $x \in R_{k}^{\prime}$. In particular, since $t_{i}^{p^{n}}=t_{i}$, we see that $f\left(t_{i}\right)=g\left(t_{i}\right)$ for all $i$, and so $f=g$. Also, given $\bar{f}: R_{k}^{\prime} \rightarrow A / N$, choose $y_{i} \in A$ such that $\bar{f}\left(t_{i}\right)=y_{i}+N$. Then define $f: R_{k}^{\prime} \rightarrow A$ by $f\left(t_{i}\right)=y_{i}^{p^{n}}$. Since $\bar{f}$ is a ring map, $y_{i}^{p^{n}}-y_{i} \in N$. Hence $\left(y_{i}^{p^{n}}\right)^{p^{n}}=y_{i}^{p^{n}}$. Similarly, since $y_{0}^{p^{n}-1}-1 \in N$, we see that $y_{0}^{p^{n+1}-p}=1$, and so $y_{0}$ is a unit. Hence $f$ is a ring map lifting $\bar{f}$.

By combining Theorem 2.8 with Theorem 3.6, Lemma 3.3, and Theorem 3.13, we get the following theorem.

Theorem 3.14. The map $\Phi: E_{*}^{\vee} E \rightarrow C\left(\Gamma, E_{*}\right)$ is an isomorphism if and only if $\operatorname{Alg}_{\mathbb{F}_{p^{n}}}\left(\Psi, \overline{\mathbb{F}_{p}}\right)$ is an isomorphism, where $\Psi: K_{0} E \rightarrow C\left(\Gamma, \mathbb{F}_{p^{n}}\right)$ is induced by $\Phi$.

\section{Geometric points}

In this section, we prove that

$$
\Phi: E_{*}^{\vee} E \rightarrow C\left(\Gamma, E_{*}\right)
$$

is an isomorphism by calculating the effect of

$$
\Psi: K_{0} E \rightarrow C\left(\Gamma, \mathbb{F}_{p^{n}}\right)
$$

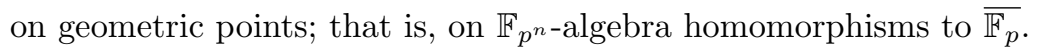

Proposition 4.1. $\operatorname{Alg}_{\mathbb{F}_{p^{n}}}\left(K_{0} E, \overline{\mathbb{F}_{p}}\right)$ is isomorphic to the set of pairs $(\alpha, \beta)$, where $\alpha$ is an automorphism of the Honda formal group law $F_{n}$ defined over $\mathbb{F}_{p^{n}}$, and $\beta$ is an element of the Galois group of $\mathbb{F}_{p^{n}}$ over $\mathbb{F}_{p}$.

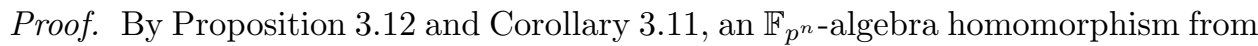
$K_{0} E$ to $\overline{\mathbb{F}_{p}}$ is equivalent to an automorphism of $F_{n}$ defined over $\overline{\mathbb{F}_{p}}$ together with a ring homomorphism $\mathbb{F}_{p^{n}} \rightarrow \overline{\mathbb{F}_{p}}$. A ring homomorphism $\mathbb{F}_{p^{n}} \rightarrow \overline{\mathbb{F}_{p}}$ is equivalent to an element of the Galois group of $\mathbb{F}_{p^{n}}$ over $\mathbb{F}_{p}$. Also, any endomorphism of $F_{n}$ over $\overline{\mathbb{F}_{p}}$ is in fact defined over $\mathbb{F}_{p^{n}}$ by [Rav86, Theorem A2.2.17].

Proposition 4.2. $\operatorname{Alg}_{\mathbb{F}_{p^{n}}}\left(C\left(\Gamma, \mathbb{F}_{p^{n}}\right), \overline{\mathbb{F}_{p}}\right)$ is isomorphic to $\Gamma$. The isomorphism takes $x \in \Gamma$ to the map that evaluates at $x$.

Proof. In view of Lemma 2.3, we have

$$
\operatorname{Alg}_{\mathbb{F}_{p^{n}}}\left(C\left(\Gamma, \mathbb{F}_{p^{n}}\right), \overline{\mathbb{F}_{p}}\right) \cong \lim _{U} \operatorname{Alg}_{\mathbb{F}_{p^{n}}}\left(F\left(\Gamma / U, \mathbb{F}_{p^{n}}\right), \overline{\mathbb{F}_{p}}\right),
$$

as $U$ runs over the open normal subgroups of $\Gamma$. But $F\left(\Gamma / U, \mathbb{F}_{p^{n}}\right)$ is generated as an

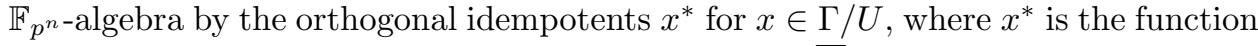
that takes $x$ to 1 and everything else to 0 . Note that $\sum_{x \in \Gamma / U} x^{*}=1$. There are no idempotents in $\overline{\mathbb{F}_{p}}$ except 0 and 1 . It follows easily that

$$
\operatorname{Alg}_{\mathbb{F}_{p^{n}}}\left(F\left(\Gamma / U, \mathbb{F}_{p^{n}}\right), \overline{\mathbb{F}_{p}}\right) \cong \Gamma / U
$$

where the isomorphism takes $x \in \Gamma / U$ to the map that evaluates at $x$. Taking inverse limits gives the desired result. 
Thus to proceed any further, we must identify the group $\Gamma$. Strickland does this in [Str00, Proposition 4], but we will fill in many missing details.

We first prove some preliminary results about Landweber exact spectra. Some of this will require some of the results of [HS99, Section 2.1], where the authors study Landweber exact spectra whose homotopy is evenly graded. Recall that, if $(A, \Gamma)$ is a Hopf algebroid and $M$ and $N$ are $\Gamma$-comodules, then $M \wedge N$ denotes the comodule which is isomorphic to the tensor product of the left $A$-modules $M$ and $N$, with the diagonal coaction of $\Gamma$.

Lemma 4.3. Suppose $F$ and $G$ are evenly graded Landweber exact spectra. Then the natural map $M U_{*} F \wedge M U_{*} G \rightarrow M U_{*}(F \wedge G)$ of $M U_{*} M U$-comodules is an isomorphism.

Proof. Any evenly graded Landweber exact spectrum is a minimal weak colimit of finite spectra that have only even-dimensional cells, by [HS99, Proposition 2.12]. Such a spectrum is called evenly generated in [HS99, Section 2.1]. The lemma at hand is true for any evenly generated spectra $F$ and $G$. Indeed, the lemma is clear for finite spectra with only even-dimensional cells, and then one takes a suitable colimit.

Lemma 4.4. Suppose $F$ and $G$ are Landweber exact spectra. Then $F \wedge G$ is also Landweber exact.

Proof. Landweber exactness implies that

$$
(F \wedge G)_{*} \cong F_{*} \otimes_{M U_{*}} M U_{*} M U \otimes_{M U_{*}} G_{*}
$$

as $M U_{*}$-modules. Now let $(A, \Gamma)$ be any Hopf algebroid. An $A$-module $B$ is called Landweber exact if $B \otimes_{A}(-)$ takes exact sequences of $\Gamma$-comodules to exact sequences of $A$-modules. This is equivalent to $B \otimes_{A} \Gamma$ being flat as a right $A$-module, by [HS03, Lemma 2.2]. Now, if $B$ and $C$ are Landweber exact, then $B \otimes_{A} \Gamma \otimes_{A} C$ is obviously Landweber exact since

$$
B \otimes_{A} \Gamma \otimes_{A} C \otimes_{A} \Gamma \cong\left(B \otimes_{A} \Gamma\right) \otimes_{A}\left(C \otimes_{A} \Gamma\right)
$$

is flat, as the tensor product of two flat modules.

Proposition 4.5. Suppose $F$ and $G$ are Landweber exact, evenly graded, commutative $M U$-algebra spectra. Let $\phi_{F}: M U_{*} F \rightarrow F_{*}$ denote the map induced by the action of $M U$ on $F$, and let $\eta_{F}: F_{*} \rightarrow M U_{*} F$ denote the map induced by the unit of $M U$.

1. The map $[F, G] \rightarrow \operatorname{Hom}_{M U_{*}}\left(M U_{*} F, G_{*}\right)$ that takes $f$ to $\phi_{G} \circ M U_{*} f$ is an isomorphism.

2. The map $f: F \rightarrow G$ is an isomorphism if and only if $\phi_{G} \circ M U_{*} f \circ \eta_{F}$ is an isomorphism.

3. The map $f: F \rightarrow G$ is a map of ring spectra if and only if $\phi_{G} \circ M U_{*} f$ is a map of $M U_{*}$-algebras. 
Proof. Part 1 is proved in [HS99, Corollary 2.17]. For part 2, we note that

$$
\phi_{G} \circ M U_{*} f \circ \eta_{F}=\phi_{G} \circ \eta_{G} \circ f_{*}=f_{*} .
$$

For part 3 , if $f$ is a map of ring spectra, then $M U_{*} f$ is a map of $M U_{*}$-algebras, so $\phi_{G} \circ M U_{*} f$ is also a map of $M U_{*}$-algebras. Conversely, suppose $\phi_{G} \circ M U_{*} f$ is a map of $M U_{*}$-algebras. Then it must take 1 to 1 , from which it follows easily that $f$ is compatible with the unit. To see that $f$ is compatible with the multiplication, we first prove that $M U_{*} f$ is a map of $M U_{*}$-algebras. Indeed, consider the diagram below.

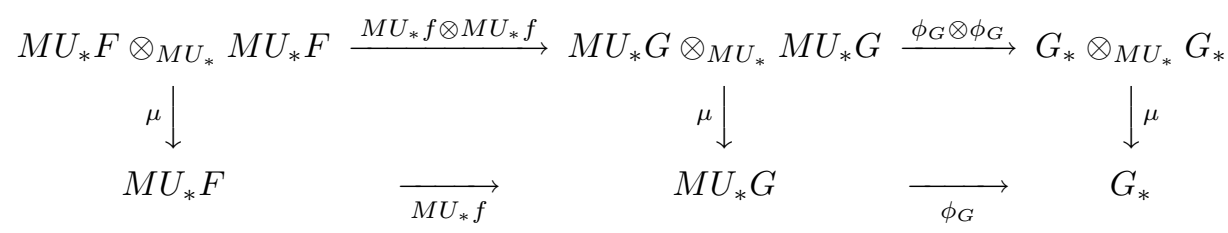

The right-hand square of this diagram is commutative. We want to show that the left-hand square is commutative. Because $M U_{*} G \cong M U_{*} M U \otimes_{M U_{*}} G_{*}$ is an extended $M U_{*} M U$-comodule, it suffices to check that the two composites become equal upon applying $\phi_{G}$, This is equivalent to checking whether the outer boundary of the diagram is commutative. But this is true since $\phi \circ M U_{*} f$ is a map of $M U_{*}$-algebras.

We have now shown that $M U_{*} f$ is a map of $M U_{*}$-algebras. Using the isomorphism of Lemma 4.3, we see that the diagram below is commutative.

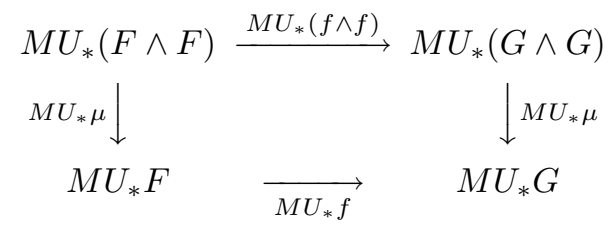

But $F \wedge F$ and $G \wedge G$ are also Landweber exact and evenly graded by Lemma 4.4. Hence we can apply part 1 in the form

$$
[F \wedge F, G] \cong \operatorname{Hom}_{M U_{*} M U}\left(M U_{*}(F \wedge F), M U_{*} G\right)
$$

to conclude that $F \rightarrow G$ is a map of ring spectra.

Corollary 4.6. Suppose $F$ is a Landweber exact, evenly graded, commutative $M U$ algebra spectra. Then the set of ring spectrum automorphisms of $F$ is isomorphic to the set of $F_{*}$-algebra homomorphisms $f: F_{*} F \rightarrow F_{*}$ such that $f \eta_{R}: F_{*} \rightarrow F_{*}$ is an isomorphism.

Proof. By Proposition 4.5, the set of ring spectrum automorphisms of $F$ is isomorphic to the set of maps of $M U_{*}$-algebras $h: M U_{*} F \rightarrow F_{*}$ such that $h \circ \eta_{E}$ is an isomorphism. This is equivalent to the set of $F_{*}$-algebra homomorphisms

$$
f: F_{*} F \cong F_{*} \otimes_{M U_{*}} M U_{*} F \rightarrow F_{*}
$$

such that $f \eta_{R}$ is an isomorphism. 
We can now apply this to our spectrum $E$. Recall that there is a $p$-typical formal group law $F$ over $E_{0}$, whose classifying map takes $v_{i}$ to $u_{i}$ for $i<n$, takes $v_{n}$ to 1 , and takes all other $v_{i}$ to 0 .

Proposition 4.7. The group $\Gamma$ is isomorphic to the set of all pairs $(\tau, \phi)$ where $\tau$ is an automorphism of the ring $E_{0}$ and $\phi$ is an isomorphism from the formal group $F$ to $\tau_{*} F$.

Proof. In view of Corollary 4.6 and the fact that $E_{*}$ has a unit $u$ in degree 2, we see that $\Gamma$ is isomorphic to the set of all $E_{0}$-algebra maps

$$
f: E_{0} E \rightarrow E_{0}
$$

such that $f \eta_{R}$ is an isomorphism. Proposition 3.8 tells us that

$$
E_{0} E \cong E_{0} \otimes_{V} V T\left[t_{0}, t_{0}^{-1}\right] \otimes_{V} E_{0},
$$

Since the tensor product is the coproduct in the category of commutative rings, and $V T\left[t_{0}, t_{0}^{-1}\right]$ is the representing ring for isomorphisms of formal group laws, we get the desired result.

Recall that the correspondence between elements of $\Gamma$ and $E_{0}$-algebra maps $f: E_{0} E \rightarrow E_{0}$ for which $f \eta_{R}$ is an isomorphism takes $\gamma$ to $\left(\pi_{0} \mu\right) \circ E_{0}(\gamma)$. The following corollary is then immediate.

Corollary 4.8. If $\gamma \in \Gamma$ corresponds to $(\tau, \phi)$ as in Proposition 4.7, then the induced map

$$
\left(\pi_{0} \mu\right) \circ E_{0}(\gamma): E_{0} E \cong E_{0} \otimes_{V} V T\left[t_{0}, t_{0}^{-1}\right] \otimes_{V} E_{0} \rightarrow E_{0}
$$

takes $a \otimes b \otimes c$ to $a s_{\phi}(b) \tau(c)$, where $s_{\phi}$ denotes the classifying map of $\phi$.

We can now identify $\Gamma$, following [Str00, Proposition 4].

Theorem 4.9. Let $\Gamma_{0}$ denote the automorphism group of the Honda formal group law $F_{n}$ as a formal group law over $\mathbb{F}_{p^{n}}$, and let $C$ denote the Galois group of $\mathbb{F}_{p^{n}}$ over $\mathbb{F}_{p}$. Then $\Gamma \cong \Gamma_{0} \rtimes C$. In particular, $\Gamma$ is isomorphic to the set of pairs $(\alpha, \beta)$, where $\alpha$ is an automorphism of $F_{n}$ defined over $\mathbb{F}_{p^{n}}$, and $\beta$ is an element of the Galois group of $\mathbb{F}_{p^{n}}$ over $\mathbb{F}_{p}$.

Proof. Proposition 4.7 and Lubin-Tate deformation theory [LT66] show that $\Gamma$ is isomorphic to the set of all pairs $(\alpha, \beta)$ where $\beta$ is an automorphism of $\mathbb{F}_{p^{n}} \cong E_{0} / \mathfrak{m}$ and $\alpha$ is an isomorphism of the reduction $F_{n}$ of $F$ modulo $\mathfrak{m}$ with $\beta_{*} F_{n}$. But $F_{n}$ is defined over $\mathbb{F}_{p}$, so $\beta_{*} F_{n}=F_{n}$. Hence $\alpha$ is an automorphism of $F_{n}$ defined over $\mathbb{F}_{p^{n}}$, and $\beta$ is an element of $C$.

There is an obvious action of $C$ on $\Gamma_{0}$; if $\sigma \in C$, then $\sigma$ induces an isomorphism $\mathbb{F}_{p^{n}}[[x]] \rightarrow \mathbb{F}_{p^{n}}[[x]]$ that takes $\sum_{i} c_{i} x^{i}$ to $\sum_{i} c_{i}^{\sigma} x^{i}$. This isomorphism preserves $\Gamma_{0}$. We leave to the reader the check that the multiplication of $\Gamma$ corresponds to the multiplication on $\Gamma_{0} \rtimes C$.

Combining Theorem 4.9 with Proposition 4.1, we see that the two sets

$$
\operatorname{Alg}_{\mathbb{F}_{p^{n}}}\left(K_{0} E, \overline{\mathbb{F}_{p}}\right) \text { and } \mathbf{A l g}_{\mathbb{F}_{p^{n}}}\left(C\left(\Gamma, \mathbb{F}_{p^{n}}\right), \overline{\mathbb{F}_{p}}\right)
$$


are abstractly isomorphic. To see that the map $\operatorname{Alg}_{\mathbb{F}_{p^{n}}}\left(\Psi, \overline{\mathbb{F}_{p}}\right)$ is an isomorphism, though, we need to understand the map $\Psi$ better. The main point is the following corollary.

Corollary 4.10. If $\gamma \in \Gamma$ corresponds to $(\alpha, \beta)$ as in Theorem 4.9, then the map

$$
\Psi: \mathbb{F}_{p^{n}} \otimes_{V} V T\left[t_{0}, t_{0}^{-1}\right] \otimes_{V} \mathbb{F}_{p^{n}} \cong K_{0} E \rightarrow C\left(\Gamma, \mathbb{F}_{p^{n}}\right)
$$

has $\Psi(a \otimes b \otimes c)(\gamma)=a s_{\alpha}(b) \beta(c)$, where $s_{\alpha}$ is the classifying map of $\alpha$.

Proof. Recall that $\Psi$ is induced by $\Phi$ by dividing by $\mathfrak{m}$ and taking the induced map in degree 0 . Using the fact that $K_{*} E=E_{*}^{\vee} E / \mathfrak{m}$ (see Lemma 2.1), we see that $\Psi(a)(\gamma)$ is the composite

$$
S^{0} \stackrel{a}{\rightarrow} K \wedge E \stackrel{1 \wedge \gamma}{\longrightarrow} K \wedge E \stackrel{\mu}{\longrightarrow} K .
$$

Since the Lubin-Tate correspondence proceeds by reducing a pair $(\tau, \phi)$ as in Proposition 4.7 modulo $\mathfrak{m}$ to obtain $(\alpha, \beta)$, this corollary follows from Corollary 4.8.

We can now complete the proof that $\Phi$ is an isomorphism.

Theorem 4.11. The map

$$
\Phi: E_{*}^{\vee} E \rightarrow C\left(\Gamma, E_{*}\right)
$$

of Section 1 is an isomorphism.

Proof. In view of Theorem 3.14, it suffices to show that $\operatorname{Alg}_{\mathbb{F}_{p^{n}}}\left(\Psi, \overline{\mathbb{F}_{p}}\right)$ is an isomorphism. By Proposition 4.2, a typical element of $\operatorname{Alg}_{\mathbb{F}_{p^{n}}}\left(C\left(\Gamma, \mathbb{F}_{p^{n}}\right), \overline{\mathbb{F}_{p}}\right)$ is the evaluation at $\gamma$ map $\mathrm{Ev}_{\gamma}$ for $\gamma \in \Gamma$. If $\gamma$ corresponds to the pair $(\alpha, \beta)$ as in Theorem 4.9, then Corollary 4.10 tells us that $\operatorname{Ev}_{\gamma} \circ \Psi$ is the $\mathbb{F}_{p^{n} \text {-algebra map }}$

$$
\operatorname{Ev}_{\gamma} \circ \Psi: \mathbb{F}_{p^{n}} \otimes_{V} V T\left[t_{0}, t_{0}^{-1}\right] \otimes_{V} \mathbb{F}_{p^{n}} \rightarrow \overline{\mathbb{F}_{p}}
$$

that takes $a \otimes b \otimes c$ to $a s_{\alpha}(b) \beta(c)$, where $s_{\alpha}$ is the classifying map of $\alpha$. Under the isomorphism of Proposition 4.1, this map corresponds to $(\alpha, \beta)$. Thus $\operatorname{Alg}_{\mathbb{F}_{p^{n}}}\left(\Psi, \overline{\mathbb{F}_{p}}\right)$ is an isomorphism.

\section{Cohomology operations}

In this section, we prove that $E^{*} E$ is isomorphic to the twisted completed group ring $E_{*}[[\Gamma]]$. The strategy is to construct the commutative square below,

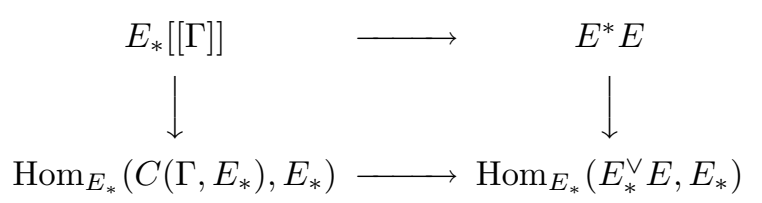

and to show that the vertical maps are isomorphisms.

We begin with the right-hand vertical map in Section 5.1, and we discuss the left-hand vertical map in Section 5.2. We then complete the proof in Section 5.3. 


\subsection{Duality for $E^{*} E$}

The object of this section is to prove the following theorem.

Theorem 5.1. Suppose $X$ is a spectrum such that $K_{*} X$ is evenly graded. Then the natural map

$$
E^{*} X \stackrel{\rho}{\rightarrow} \operatorname{Hom}_{E_{*}}\left(E_{*}^{\vee} X, E_{*}\right)
$$

is an isomorphism.

The natural map in question takes an element $x \in E^{m} X$ to the homomorphism that takes $y \in E_{k}^{\vee} X$ to the composite

$$
S^{k-m} \stackrel{\Sigma^{-m} y}{\longrightarrow} E \widetilde{\wedge} \Sigma^{-m} X \stackrel{1 \widetilde{\wedge} x}{\longrightarrow} E \widetilde{\wedge} E \stackrel{\mu}{\longrightarrow} E .
$$

One can define an analogous map

$$
\rho: F^{*} X \rightarrow \operatorname{Hom}_{E_{*}}\left(E_{*}^{\vee} X, F_{*}\right)
$$

for any $E$-module spectrum $F$.

Note that Theorem 5.1 would be automatic if we had a suitable universal coefficient spectral sequence for $E_{*}^{\vee}(-)$. Indeed, since $K_{*} X$ is evenly graded, $E_{*}^{\vee} X$ is pro-free and so projective in the category of $L$-complete $E_{*}$-modules $[\mathbf{H S 9 9}$, Theorem A.9]. We do have a universal coefficient spectral sequence for $E_{*}(-)$, following Adams' approach [Ada74, Section III.13]. But our hypotheses do not guarantee that $E_{*} X$ is projective over $E_{*}$. A universal coefficient spectral sequence for $E_{*}^{\vee}(-)$ has in fact recently been constructed [Hov04].

We will prove Theorem 5.1 by working with $(E / J)^{*}(X)$, where

$$
J=\left(p^{i_{0}}, u_{1}^{i_{1}}, \ldots, u_{n-1}^{i_{n-1}}\right)
$$

is a regular ideal in $E_{*}$, and $E / J$ is obtained by taking successive cofibers as in [EKMM97, Chapter V]. The ring $E_{*} / J$ is a local ring with nilpotent maximal ideal $\mathfrak{m}$. We have the following lemma, which is surely well-known, about modules over such rings.

Lemma 5.2. Suppose $f: M \rightarrow N$ is a map of $R$-modules, where $R$ is a local ring with nilpotent maximal ideal $\mathfrak{m}$.

1. $f$ is surjective if and only if $R / \mathfrak{m} \otimes_{R} f$ is surjective.

2. If $M$ and $N$ are flat and $R / \mathfrak{m} \otimes_{R} f$ is an isomorphism, then $f$ is an isomorphism.

It follows easily from this lemma that flat $R$-modules are in fact free, though we do not need this fact.

Proof. Suppose that $R / \mathfrak{m} \otimes_{R} f$ is surjective. Given $y$ in $N$, we show by induction on $k$ that there is an $x_{k}$ in $M$ such that $f\left(x_{k}\right)-y \in \mathfrak{m}^{k} N$. Since $\mathfrak{m}$ is nilpotent, taking $k$ large enough shows that $f$ is surjective. The case $k=1$ is clear since $R / \mathfrak{m} \otimes_{R} f$ is surjective. Now suppose that

$$
f\left(x_{k}\right)-y=r_{1} z_{1}+\cdots+r_{j} z_{j}
$$


where $r_{i} \in \mathfrak{m}^{k}$. For each $i$, we can find an $w_{i} \in M$ such that $z_{i}=f\left(w_{i}\right)+t_{i}$, where $t_{i} \in \mathfrak{m} N$, by the base case of the induction. It follows that

$$
f\left(x_{k}-r_{1} w_{1}-\cdots-r_{j} w_{j}\right)-y \in \mathfrak{m}^{k+1} N
$$

proving the induction step.

Now suppose $M$ and $N$ are flat. Let $K$ denote the kernel of $f$. Since $N$ is flat, the sequence

$$
0 \rightarrow K \rightarrow M \stackrel{f}{\rightarrow} N \rightarrow 0
$$

pure, and so $K$ is flat [Lam99, Corollary 4.86] and $K / \mathfrak{m} K=0$. Since $\mathfrak{m}^{k} / \mathfrak{m}^{k+1}$ is a free module over the field $R / \mathfrak{m}$, we see that $K \otimes_{R} \mathfrak{m}^{k} / \mathfrak{m}^{k+1}=0$ for all $k$. Tensoring the short exact sequence

$$
0 \rightarrow \mathfrak{m}^{k} / \mathfrak{m}^{k+1} \rightarrow R / \mathfrak{m}^{k+1} \rightarrow R / \mathfrak{m}^{k} \rightarrow 0
$$

with $K$, we conclude by induction that $K / \mathfrak{m}^{k} K=0$ for all $k$. Since $\mathfrak{m}$ is nilpotent, we see that $K=0$.

With this lemma in hand, we can now analyse $(E / J)^{*}(X)$.

Proposition 5.3. Suppose $X$ is a spectrum such that $K_{*} X$ is evenly graded, and $J=\left(p^{i_{0}}, u_{1}^{i_{1}}, \ldots, u_{n-1}^{i_{n-1}}\right)$ is a regular ideal in $E_{*}$. Then the natural map

$$
\rho:(E / J)^{*}(X) \rightarrow \operatorname{Hom}_{E_{*}}\left(E_{*}^{\vee} X, E_{*} / J\right)
$$

is an isomorphism.

Proof. Note that

$$
\operatorname{Hom}_{E_{*}}\left(E_{*}^{\vee} X, E_{*} / J\right) \cong \operatorname{Hom}_{E_{*} / J}\left(\left(E_{*}^{\vee} X\right) / J, E_{*} / J\right) .
$$

and $E_{*} / J$ is a commutative ring with nilpotent maximal ideal $\mathfrak{m}=\left(p, u_{1}, \ldots, u_{n-1}\right)$. The plan is thus to use Lemma 5.2.

Since $K_{*} X$ is evenly graded, so is its dual $K^{*} X$. Thus $E^{*} X$ is pro-free by Lemma 2.1. In particular, the sequence $\left(p, u_{1}, \ldots, u_{n-1}\right)$ is a regular sequence on $E^{*} X$. It follows from [Mat89, Theorem 16.1] that

$$
\left(p^{i_{0}}, u_{1}^{i_{1}}, \ldots, u_{n-1}^{i_{n-1}}\right)
$$

is also a regular sequence on $E^{*} X$, and therefore that $(E / J)^{*} X \cong\left(E^{*} X\right) / J$, Since $E^{*} X$ is the completion of a free $E_{*}$-module, we conclude that $(E / J)^{*} X$ is a free $E_{*} / J$-module, and that $\left((E / J)^{*} X\right) / \mathfrak{m} \cong K^{*} X$.

Similarly, because $K_{*} X$ is evenly graded, $E_{*}^{\vee} X$ is evenly graded and pro-free by Lemma 2.1. The same argument as in the preceding paragraph then shows that

$$
(E / J)_{*} X \cong\left(E_{*}^{\vee} X\right) / J
$$

and is a free $E_{*} / J$-module. It follows that $\operatorname{Hom}_{E_{*} / J}\left(\left(E_{*}^{\vee} X\right) / J, E_{*} / J\right)$ is a product of free modules, and so is flat (since $E_{*} / J$ is Noetherian). It also follows that $\left((E / J)_{*} X\right) / \mathfrak{m} \cong K_{*} X$

Furthermore, there is a natural map

$$
\operatorname{Hom}_{E_{*} / J}\left(\left(E_{*}^{\vee} X\right) / J, E_{*} / J\right) \rightarrow \operatorname{Hom}_{E_{*} / J}\left(\left(E_{*}^{\vee} X\right) / J, K_{*}\right) \cong \operatorname{Hom}_{K_{*}}\left(K_{*} X, K_{*}\right) .
$$


This map is surjective, since $\left(E_{*}^{\vee} X\right) / J$ is free. Hence we get an induced surjection

$$
\left(\operatorname{Hom}_{E_{*} / J}\left(\left(E_{*}^{\vee} X\right) / J, E_{*} / J\right)\right) / \mathfrak{m} \rightarrow \operatorname{Hom}_{K_{*}}\left(K_{*} X, K_{*}\right) .
$$

We claim this map is an isomorphism. To see this suppose $f:\left(E_{*}^{\vee} X\right) / J \rightarrow E_{*} / J$ is a homomorphism that goes to zero as a map to $K_{*}$. This means that $f(x) \in \mathfrak{m}$ for all $x$. In particular, if we let $\left\{e_{i}\right\}$ be a generating set for the free module $\left(E_{*}^{\vee} X\right) / J$, we can write

$$
f\left(e_{i}\right)=p x_{i 0}+u_{1} x_{i 1}+\cdots u_{n-1} x_{i, n-1}
$$

for some elements $x_{i j}$ in $E_{*} / J$. But then we can define homomorphisms

$$
g_{j}:\left(E_{*}^{\vee} X\right) / J \rightarrow E_{*} / J
$$

for $j=0,1, \ldots, n-1$ by $g_{j}\left(e_{i}\right)=x_{i j}$. This gives

$$
f=p g_{0}+u_{1} g_{1}+\cdots u_{n-1} g_{n-1},
$$

and so $f \in \mathfrak{m} \operatorname{Hom}_{E_{*} / J}\left(\left(E_{*}^{\vee} X\right) / J, E_{*} / J\right)$, as required.

It now follows from an easy diagram chase that if we tensor the map

$$
(E / J)^{*} X \rightarrow \operatorname{Hom}_{E_{*}}\left(E_{*}^{\vee} X, E_{*} / J\right)
$$

with $E_{*} / \mathfrak{m}$, we get the isomorphism

$$
K^{*} X \cong \operatorname{Hom}_{K_{*}}\left(K_{*} X, K_{*}\right) .
$$

Lemma 5.2 completes the proof.

We now prove Theorem 5.1.

Proof of Theorem 5.1. Choose a sequence of ideals

$$
\cdots \subseteq J_{k} \subseteq J_{k-1} \subseteq \cdots \subseteq J_{1}
$$

such that each $J_{k}$ is of the form $\left(p^{i_{0}}, u_{1}^{i_{1}}, \ldots, u_{n-1}^{i_{n-1}}\right)$ and the $J_{k}$ converge to 0 in the $\mathfrak{m}$-adic topology. We have a commutative square

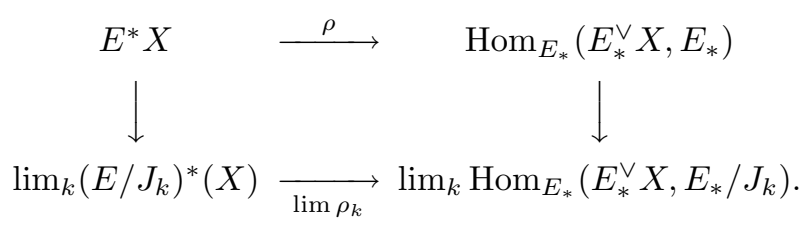

The right-hand vertical map is an isomorphism because $E_{*}$ is complete. To understand the left-hand vertical map, we apply the Milnor exact sequence

$$
0 \rightarrow \lim ^{1}\left(E / J_{k}\right)^{*+1} X \rightarrow E^{*} X \rightarrow \lim \left(E / J_{k}\right)^{*} X \rightarrow 0 .
$$

Since $K_{*} X$ is evenly graded, $\left(E / J_{k}\right)^{*} X \cong\left(E_{*}^{\vee} X\right) / J_{k}$ as we have seen in the proof of Proposition 5.3. Thus the $\lim ^{1}$-terms vanish, and so the left-hand vertical map is an isomorphism. Proposition 5.3 implies that the bottom horizontal map is an isomorphism as well, completing the proof. 


\subsection{Duality for $C(G, R)$}

We now turn our attention to the twisted completed group ring $E_{*}[[\Gamma]]$.

In general, whenever $G$ is a profinite group and $\mathfrak{a}$ is an ideal in a ring $R$, we can define the $R$-module

$$
R[[G]]=\lim _{k} \lim _{U}\left(R / \mathfrak{a}^{k}\right)[G / U] .
$$

We then have the following theorem.

Theorem 5.4. Let $G$ be a profinite group and $\mathfrak{a}$ an ideal in a commutative ring $R$ such that $R$ is complete in the $\mathfrak{a}$-adic topology. Then the natural map

$$
R[[G]] \rightarrow \operatorname{Hom}_{R}(C(G, R), R)
$$

is an isomorphism of $R$-modules.

Proof. There is a natural map

$$
\alpha: R[G] \rightarrow \operatorname{Hom}_{R}(C(G, R), R),
$$

where $\alpha(r[g])(f)=r f(g)$. It is clear that $\alpha$ is an isomorphism when $G$ is finite. Hence we get an induced natural isomorphism

$$
R[[G]]=\lim _{k, U}\left(R / \mathfrak{a}^{k}\right)[G / U] \rightarrow \lim _{k, U} \operatorname{Hom}_{R / \mathfrak{a}^{k}}\left(C\left(G / U, R / \mathfrak{a}^{k}\right), R / \mathfrak{a}^{k}\right) .
$$

We must show that the right-hand side of this isomorphism is naturally isomorphic to $\operatorname{Hom}_{R}(C(G, R), R)$. To see this, we first use Proposition 2.4 and Proposition 2.5 to conclude that $C(G, R) / \mathfrak{a}^{k} \cong C\left(G, R / \mathfrak{a}^{k}\right)$ This fact together with Lemma 2.3 gives us the following chain of isomorphisms

$$
\begin{gathered}
\operatorname{Hom}_{R}(C(G, R), R) \cong \lim _{k} \operatorname{Hom}_{R}\left(C(G, R), R / \mathfrak{a}^{k}\right) \\
\cong \lim _{k} \operatorname{Hom}_{R / \mathfrak{a}^{k}}\left(C\left(G, R / \mathfrak{a}^{k}\right), R / \mathfrak{a}^{k}\right) \\
\cong \lim _{k} \lim _{U} \operatorname{Hom}_{R / \mathfrak{a}^{k}}\left(C\left(G / U, R / \mathfrak{a}^{k}\right), R / \mathfrak{a}^{k}\right),
\end{gathered}
$$

completing the proof.

Now, when $G$ acts continuously on $R, \operatorname{Hom}_{R}(C(G, R), R)$ is the dual of the Hopf algebroid $C(G, R)$, so is an $R$-algebra. In view of Theorem 5.4, then, $R[[G]]$ should also be an algebra, in analogy with the group ring, but in a way that takes into account the action of $G$ on $R$.

To see how this works, assume first that an arbitrary group $G$ acts on a commutative ring $R$ by ring isomorphisms. Then the twisted group $\operatorname{ring} R[G]$ is the free $R$-module generated by the elements of $G$, with multiplication defined by $(a[g])(b[h])=a b^{g}[g h]$. We would like to realize $R[[G]]$ as a completion of the twisted group ring $R[G]$ with respect to a suitable family of ideals. For this, we need to assume $G$ is a profinite group, $R$ is a local ring with maximal ideal $\mathfrak{m}$ that is complete in the m-adic topology, and $G$ acts continuously on $R$, and, even better, acts through a finite quotient on $R / \mathfrak{m}^{k}$ for all $k$. That is, we need to assume that, for all $k$, there is an open normal subgroup $U_{k}$ of $G$ such that $U_{k}$ acts trivially on $R / \mathrm{m}^{k}$. Note that this is automatic from continuity if $R / \mathfrak{m}^{k}$ is finite, or, in the graded case, 
finite in each degree, as is true for $R=E_{*}$. Indeed, in this case $\operatorname{Aut}\left(R / \mathfrak{m}^{k}\right)$ is finite, so the homomorphism $G \rightarrow \operatorname{Aut}\left(R / \mathfrak{m}^{k}\right)$ must factor through a finite quotient.

Assuming that $G$ does act through a finite quotient on each $R / \mathfrak{m}^{k}$, we define ideals $I(k, U)$ of $R[G]$ for each integer $k$ and each open normal subgroup $U$ of $G$ such that $U$ acts trivially on $R / \mathrm{m}^{k}$. The ideal $I(k, U)$ is the kernel of the surjection $R[G] \rightarrow\left(R / \mathfrak{m}^{k}\right)[G / U]$ to the twisted group ring. In more concrete terms, $I(k, U)$ is the set of all elements $\sum a_{g}[g]$ in $R[G]$ such that for every coset $C$ of $U$ in $G$, we have $\sum_{g \in C} a_{g} \in \mathfrak{m}^{k}$. We then the twisted completed group ring $R[[G]]$ to be the completion of $R[G]$ with respect to the ideals $I(k, U)$, so that

$$
R[[G]]=\lim _{k, U}\left(R / \mathfrak{a}^{k}\right)[G / U] .
$$

This is the same $R$-module as we defined above, since we can take this inverse limit over arbitrary pairs $(k, U)$. Note that the natural map $R[G] \rightarrow R[[G]]$ is injective since $\bigcap_{k} \mathfrak{m}^{k}=0$. All the statements above work when $R$ is a graded ring, as long as $\mathfrak{m}$ is homogeneous and the action of $G$ preserves the grading.

With these definitions, the isomorphism of Theorem 5.4 is in fact an isomorphism of $R$-algebras, using the mulitplication on $\operatorname{Hom}_{R}(C(G, R), R)$ dual to the Hopf algebroid structure on $C(G, R)$. We do not need this result, so we leave the proof to the interested reader.

\subsection{The isomorphism}

We can now compute $E^{*} E$.

Theorem 5.5. The inclusion $\Gamma \rightarrow E^{0} E$ induces an $E_{*}$-algebra isomorphism

$$
E_{*}[[\Gamma]] \rightarrow E^{*} E .
$$

Proof. There is a map $E_{*} \rightarrow E^{*} E$ that takes $r \in E_{k}$ to $\widetilde{r} \in E^{k} E$, where $\widetilde{r}$ is the composite

$$
E=S^{0} \wedge E \stackrel{r \wedge 1}{\longrightarrow} \Sigma^{k} E \wedge E \stackrel{\Sigma^{k} \mu}{\longrightarrow} \Sigma^{k} E .
$$

The inclusion of $\Gamma$ then certainly induces a map $\alpha: E_{*}[\Gamma] \rightarrow E^{*} E$, defined by letting $\alpha(r[\gamma])$ be the composite $\widetilde{r} \circ \gamma$. To see that $\alpha$ is an $E_{*}$-algebra homomorphism, use the commutative diagram below.

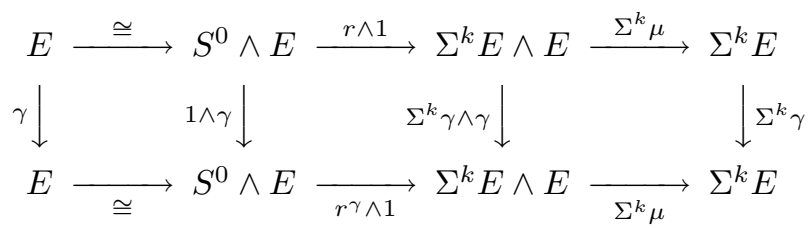

This diagram commutes because $\gamma$ is a map of ring spectra, and it shows that $\alpha([\gamma] r)=(\alpha[\gamma])(\alpha r)$, so that $\alpha$ is an $E_{*}$-algebra homomorphism.

Now, $E^{*} E$ is complete with respect to the m-adic topology, so to show that $\alpha$ extends to an $E_{*}$-algebra homomorphism

$$
\beta: E_{*}[[\Gamma]] \rightarrow E^{*} E,
$$


it suffices to show that $\alpha$ is continuous. That is, given $k$, we must find an $m$ and $U$ such that $\alpha I(m, U) \subseteq \mathfrak{m}^{k} E^{*} E$. But the action of $\Gamma$ on $E^{*} E$ is continuous, so there is a $U$ such that $U$ preserves $\mathfrak{m}^{k} E^{*} E$. This means that the composite

$$
E_{*}[\Gamma] \stackrel{\alpha}{\rightarrow} E^{*} E \rightarrow\left(E^{*} E\right) / \mathfrak{m}^{k}
$$

factors through $\left(E_{*} / \mathfrak{m}^{k}\right)[\Gamma / U]$, and so $\alpha I(k, U) \subseteq \mathfrak{m}^{k} E^{*} E$, as required.

Now consider the diagram below.

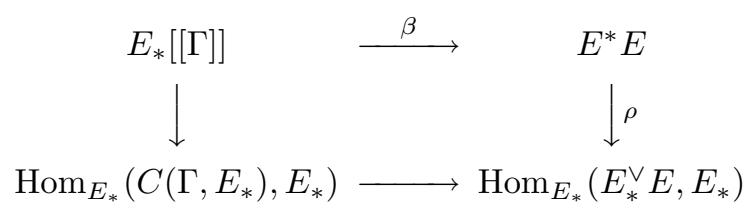

The left-hand vertical map is the isomorphism of Theorem 5.4, the right-hand vertical map is the isomorphism of Theorem 5.1, and the bottom horizontal map is dual to the isomorphism of Theorem 4.11. One can easily check that the diagram is commutative. Indeed, it suffices to check that the element $[\gamma] \in E_{*}[\Gamma]$ goes to the same place under both composites. In fact, it goes to the map that takes $x \in E_{k}^{\vee} E$ to the composite

$$
S^{0} \stackrel{x}{\longrightarrow} \Sigma^{k} E \widetilde{\wedge} E \stackrel{\Sigma^{k}(1 \widetilde{\wedge} \gamma)}{\longrightarrow} \Sigma^{k} E \widetilde{\wedge} E \stackrel{\Sigma^{k} \mu}{\longrightarrow} \Sigma^{k} E .
$$

Hence $\beta$ is an isomorphism.

\section{Hopf algebroid structure}

The object of this section is to show that $\left(E_{*}, E_{*}^{\vee} E\right)$ and $\left(E_{*}, C\left(\Gamma, E_{*}\right)\right)$ are both graded formal Hopf algebroids, and that the isomorphism $\Phi$ of Theorem 4.11 preserves the Hopf algebroid structure. We begin by defining precisely what we mean by a graded formal Hopf algebroid. We then show that $\left(E_{*}, E_{*}^{\vee} E\right)$ is a graded formal Hopf algebroid in Section 6.2, then discuss $C\left(\Gamma, E_{*}\right)$ in Section 6.3. We show that $\Phi$ preserves the Hopf algebroid structure in Section 6.4.

\subsection{Graded formal Hopf algebroids}

A graded formal Hopf algebroid should be a cogroupoid object in the category of graded formal rings. Recall from [Str99, Section 4] that a formal ring $A$ is a topological commutative ring $A$ such that cosets of open ideals form a basis for the topology on $A$, and such that $A \cong \lim A / I$ as $I$ runs through the open ideals. Morphisms in the category of formal rings are continuous homomorphisms. The category of formal rings has all finite colimits. The pushout $S \widehat{\otimes}_{R} T$ of two continuous homomorphisms $R \rightarrow S$ and $R \rightarrow T$ is the completion of the tensor product $S \otimes_{R} T$ with respect to the topology defined by the ideals $I \otimes_{R} T+S \otimes_{R} J$, where $I$ and $J$ run through open ideals in $S$ and $T$. Note that this definition of $\widehat{\otimes}$ does not conflict with our previous use of the notation, in Proposition 2.5. We can thus define a formal Hopf algebroid to be a cogroupoid object in the category of formal rings. The major difference between a formal Hopf algebroid $(A, \Gamma)$ and a Hopf algebroid 
is that the diagonal has the form $\Delta: \Gamma \rightarrow \Gamma \widehat{\otimes} \Gamma$, and so all diagrams involving $\Delta$ must be similarly changed.

Some subtleties arise in the graded case. We define a graded formal ring to be a graded ring $A$ equipped with a family $\left\{I_{j}\right\}$ of homogeneous ideals such that for all $j, j^{\prime}$ there is a $j^{\prime \prime}$ such that $I_{j^{\prime \prime}} \subseteq I_{j} \cap I_{j^{\prime}}$ and such that $A$ is the inverse limit, in the category Rings $\mathbf{~}_{g r}$ of graded rings, of the $A / I_{j}$. This means that $A_{k} \cong \lim _{j}\left(A / I_{j}\right)_{k}$ for all $k$. The family of ideals $\left\{I_{j}\right\}$ will sometimes be referred to as the topology on $A$. Maps of graded formal rings $R \rightarrow S$ are maps of graded rings such that each $R_{k} \rightarrow S_{k}$ is continuous (in the inverse limit topology).

Note that the forgetful functor from Rings $g r$ to Rings does NOT preserve inverse limits. In particular, if $k$ is a field and $x$ has degree 2, the ring $k[x]$ is a graded formal ring in the $x$-adic topology, but it is not a formal ring in the $x$-adic topology.

Nevertheless, the category of graded formal rings has all finite colimits, where the pushout of $R \rightarrow S$ and $R \rightarrow T$ is $S \widehat{\otimes}_{R} T$, the completion in the graded sense of the graded ring $S \otimes_{R} T$ with respect to the ideals $I \otimes T+S \otimes J$, where $I$ and $J$ are the homogeneous ideals that define the formal structure on $R$ and $S$. Hence we can define a graded formal Hopf algebroid to be a cogroupoid object in the category of graded formal rings.

From an algebro-geometric perspective, it would be much more natural to consider formal graded rings, rather than graded formal rings. Here a formal graded ring would be a formal ring $R$ equipped with a coaction of the Hopf algebra $\mathbb{Z}\left[u, u^{-1}\right]$. That is, the "grading" would actually be a continuous ring homomorphism $R \rightarrow R \widehat{\otimes} \mathbb{Z}\left[u, u^{-1}\right]$ that is coassociative and counital. The drawback of this approach for us is that this is just not how we think of completions in algebraic topology. For example, when we form $\left(v_{n}^{-1} B P_{*}\right)_{I_{n}}$, we do not allow the element $\sum_{k=1}^{\infty} v_{1}^{k}$. It is possible that it would be better to allow such elements, giving us homotopy groups that are only graded in this weak sense. We will stick with graded formal rings though.

\section{2. $\quad E_{*}^{\vee} E$ as a graded formal Hopf algebroid}

We now show that $\left(E_{*}, E_{*}^{\vee} E\right)$ is a graded formal Hopf algebroid. The topology is defined by the powers of the maximal ideal $\mathfrak{m}$ of $E_{*}$. We have seen that $E_{*}^{\vee} E$ is pro-free in Proposition 2.2, and so $E_{*}^{\vee} E$ is a graded formal ring. The usual proof that $\left(R_{*}, R_{*} R\right)$ is a Hopf algebroid [Rav86, Proposition 2.2.8] depends on knowing that the natural map

$$
R_{*} R \otimes_{R_{*}} R_{*} R \rightarrow R_{*}(R \wedge R)
$$

is an isomorphism. If we are to apply the same argument in this case, we need to know that

$$
E_{*}^{\vee} E \widehat{\otimes}_{E_{*}} E_{*}^{\vee} E \rightarrow E_{*}^{\vee}(E \widetilde{\wedge} E)
$$

is an isomorphism. 
Proposition 6.1. Suppose $X$ is a spectrum such that $K_{*} X$ is concentrated in even dimensions. Then the natural map

$$
\sigma:\left(E_{*}^{\vee} E \otimes_{E_{*}} E_{*}^{\vee} X\right)_{\mathfrak{m}}^{\wedge} \rightarrow E_{*}^{\vee}(E \widetilde{\wedge} X)
$$

is an isomorphism.

Proof. Since $K$ is a field spectrum, the natural map

$$
\rho: K_{*} E \otimes_{K_{*}} K_{*} X \rightarrow K_{*}(E \wedge X)=K_{*}(E \widetilde{\wedge} X)
$$

is an isomorphism. In particular, $K_{*}(E \widetilde{\wedge} X)$ is concentrated in even dimensions. Lemma 2.1 then guarantees that $E_{*}^{\vee} X$ and $E_{*}^{\vee}(E \widetilde{\wedge} X)$ are pro-free, as is $E_{*}^{\vee} E$. If $M \cong F_{\mathfrak{m}}^{\wedge}$ and $N \cong\left(F^{\prime}\right)_{\mathfrak{m}}^{\wedge}$ are pro-free modules, then $(M \otimes N)_{\mathfrak{m}}^{\wedge}$ is also pro-free, since it is isomorphic to the completion of $F \otimes_{E_{*}} F^{\prime}$. Hence $\left(E_{*}^{\vee} E \otimes_{E_{*}} E_{*}^{\vee} X\right)_{\mathfrak{m}}^{\wedge}$ is pro-free as well. In view of Lemma 2.7, to prove the proposition it suffices to check that $\sigma / \mathfrak{m}$ is an isomorphism. Using Lemma 2.1 and the fact that $\mathfrak{m}$ is an invariant ideal in $E_{*} E$, we conclude that $\sigma / \mathfrak{m} \cong \rho$, and so $\sigma$ is an isomorphism.

Corollary 6.2. Suppose $X$ is a ring spectrum such that $K_{*} X$ is concentrated in even dimensions. Then the natural map

$$
E_{*}^{\vee} X \widehat{\otimes}_{E_{*}} E_{*}^{\vee} X \rightarrow E_{*}^{\vee}(E \widetilde{\wedge} X)
$$

is an isomorphism.

Proof. By Lemma 2.1, $E_{*}^{\vee} X$ is pro-free, and so in particular is a formal ring with respect to the ideals $\mathfrak{m}^{n}$. The corollary then follows from Proposition 6.1 because $\mathfrak{m}$ is an invariant ideal in $E_{*}$.

Recall that if $R$ is a flat commutative ring spectrum, then $\left(R_{*}, R_{*} R\right)$ is a graded Hopf algebroid [Rav86, Proposition 2.2.8]. The only use of the flatness of $R$ in this argument is in the isomorphisms

$$
R_{*} X \otimes_{R_{*}} R_{*} Y \rightarrow R_{*}(X \wedge Y)
$$

in case $X=Y=R$, needed to define the diagonal, and in case $X=R, Y=R \wedge R$ and $X=R \wedge R, Y=R$, needed to prove coassociativity. We can repeat this argument using the isomorphisms of Corollary 6.2 to deduce the following theorem,

Theorem 6.3. $\left(E_{*}, E_{*}^{\vee} E\right)$ is a graded formal Hopf algebroid.

\section{3. $C\left(\Gamma, E_{*}\right)$ as a graded formal Hopf algebroid}

The object of this section is to show that $\left(E_{*}, C\left(\Gamma, E_{*}\right)\right)$ is a graded formal Hopf algebroid. We will work more generally with a profinite group $G$ acting continuously on a graded ring $R$ that is complete in the $\mathfrak{a}$-adic topology, where $\mathfrak{a}$ is a homogeneous ideal.

We begin with a very simple case. Given a group $G$, the constant group scheme $G$ is the functor on commutative rings defined by $G(S)=C(\operatorname{Spec} S, G)$. If $S$ has no nontrivial idempotents, then Spec $S$ is connected, and so $G(S)=G$, consisting only of the constant maps. The constant group scheme $G$ is in fact the coproduct in 
the category of schemes of $|G|$-many copies of Spec $\mathbb{Z}$. In particular, it is not affine when $G$ is infinite, because it is not quasi-compact.

However, when $G$ is finite, we have the following well-known proposition.

Proposition 6.4. Suppose $G$ is a finite group. Then the constant group scheme $G$ is affine and represented by the Hopf algebra $F(G, \mathbb{Z})$ of functions from $G$ to $\mathbb{Z}$.

This proposition follows from the fact that $F(G, \mathbb{Z})$ is the product of $|G|$-many copies of $\mathbb{Z}$. The structure maps of the Hopf algebra $F(G, \mathbb{Z})$ are defined as follows. The counit $\epsilon: F(G, \mathbb{Z}) \rightarrow \mathbb{Z}$ is evaluation at the identity element $e$ of $G$, the conjugation $\chi$ is induced by the inverse map in $G$, and the diagonal $\Delta$ is the composite

$$
F(G, \mathbb{Z}) \rightarrow F(G \times G, \mathbb{Z}) \stackrel{\tau_{G}^{-1}}{\longrightarrow} F(G, \mathbb{Z}) \otimes F(G, \mathbb{Z})
$$

where the first map is induced by the multiplication map of $G$ and $\tau_{G}$ is the isomorphism defined by $\tau_{G}\left(f \otimes f^{\prime}\right)(g, h)=f(g) f^{\prime}(h)$.

We can generalize Proposition 6.4 to profinite groups as well.

Proposition 6.5. Suppose $G$ is a profinite group. Then the group-valued functor on commutative rings defined by $G(S)=C(\operatorname{Spec} S, G)$, where $G$ is given the profinite topology, is an affine group scheme represented by the Hopf algebra $C(G, \mathbb{Z})$ of continuous functions from $G$ to $\mathbb{Z}$.

Note that if $S$ has no nontrivial idempotents, then $G(S)=G$, just as for the constant group scheme. There is a natural map from the constant group scheme to this profinite group scheme $G$, because every map that is continuous to the discrete topology on $G$ is also continuous to the profinite topology, and this map is an isomorphism on every $S$ with no nontrivial idempotents. But it is not an isomorphism on all $S$ unless $G$ is finite, since the constant group scheme is not affine when $G$ is infinite but the profinite group scheme is, according to Proposition 6.5.

Proof. We simply compute

$$
\begin{gathered}
G(S)=C(\operatorname{Spec} S, G) \cong \lim _{U} C(\operatorname{Spec} S, G / U) \\
\cong \lim _{U} \operatorname{Rings}(F(G / U, \mathbb{Z}), S) \cong \operatorname{Rings}(C(G, \mathbb{Z}), S) .
\end{gathered}
$$

This shows that $G$ is affine and represented by $C(G, \mathbb{Z})$. Since $G$ is visibly a groupvalued functor, $C(G, \mathbb{Z})$ is a Hopf algebra.

Note that $C(G, \mathbb{Z})$ is the colimit of the Hopf algebras $F(G / U, Z)$. One can describe the structure maps in a similar fashion. The counit is again evaluation at $e$, the conjugation is induced by the (continuous) inverse mapping on $G$, and the diagonal is the composite

$$
C(G, \mathbb{Z}) \rightarrow C(G \times G, \mathbb{Z}) \stackrel{\tau_{G}^{-1}}{\longrightarrow} C(G, \mathbb{Z}) \otimes C(G, \mathbb{Z}),
$$

where $\tau_{G}$ is again defined by $\tau_{G}\left(f \otimes f^{\prime}\right)(g, h)=f(g) f^{\prime}(h)$. The map $\tau_{G}$ is the colimit of the isomorphisms $\tau_{G / U}$, so is an isomorphism.

We now show how to build the Hopf algebroid $(R, C(G, R))$ from the Hopf algebra $C(G, \mathbb{Z})$. Recall that a Hopf algebroid is a cogroupoid object in the category 
of commutative rings [Rav86, Appendix 1]. In general, a cogroupoid object in a category $\mathcal{C}$ is a pair of objects $(A, \Gamma)$ such that $(\mathcal{C}(A, X), \mathcal{C}(\Gamma, X))$ is a groupoid that is natural in $X$ for all objects $X$ of $\mathcal{C}$. Here $\mathcal{C}(A, X)$ is the object set of the groupoid and $\mathcal{C}(\Gamma, X)$ is the morphism set. When $\mathcal{C}$ has finite colimits, $(A, \Gamma)$ is a cogroupoid object if and only if there are structure maps $\eta_{L}, \eta_{R}: A \rightarrow \Gamma, \epsilon: \Gamma \rightarrow A, \chi: \Gamma \rightarrow \Gamma$, and $\Delta: \Gamma \rightarrow \Gamma \amalg_{A} \Gamma$ analogous to the structure maps of a Hopf algebroid [Rav86, Appendix 1], where $\Gamma \amalg_{A} \Gamma$ denotes the pushout of $\Gamma \stackrel{\eta_{R}}{\longleftarrow} A \stackrel{\eta_{L}}{\longrightarrow} \Gamma$. These structure maps are required to satisfy certain axioms analogous to those of $[\operatorname{Rav86}$, Definition A1.1.1].

One of these axioms deserves special attention, because Ravenel's description of it is not optimal. Define $\Gamma \amalg_{A} \widetilde{\Gamma}$ to be the pushout where $A$ acts on the right factor of $\Gamma$ though $\eta_{R}$ instead of $\eta_{L}$; simmilarly, define $\widetilde{\Gamma} \amalg_{A} \Gamma$ to be the pushout where $A$ acts on the left factor through $\eta_{L}$ instead of $\eta_{R}$. Then axiom (f) of Ravenel's definition [Rav86, A1.1.1], which implies that the composition of a map and its inverse is the appropriate identity, should be rephrased to say that the following diagram, and a similar diagram involving $\eta_{L}$ and $1 \amalg \chi$, are commutative.

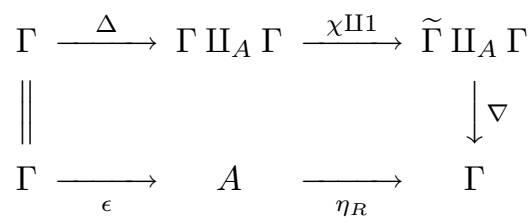

Here $\nabla$ denotes the fold map, which is the identity on each factor of $\Gamma$.

Here is a general result on constructing cogroupoid objects from actions of cogroups.

Proposition 6.6. Suppose $\mathcal{C}$ is a category with finite colimits and $C$ is a cogroup object of $\mathcal{C}$ that coacts on the right on an object $A$ of $\mathcal{C}$. Then $(A, A \amalg G)$ is a cogroupoid object of $\mathcal{C}$.

A cogroupoid object of this form is sometimes called a split cogroupoid. In the case of Hopf algebroids, this recovers the definition of a split Hopf algebroid given in [Rav86, Definition A1.1.22].

Proof. Let 0 denote the initial object of $\mathcal{C}$. Since $C$ is a cogroup, it comes with maps $\Delta: C \rightarrow C \amalg C, \epsilon: C \rightarrow 0$, and $\chi: C \rightarrow C$ playing the role of the diagonal, counit, and conjugation. There is also a coassociative and counital map $\psi: A \rightarrow A \amalg C$ giving the coaction of $C$. We then define the left unit $\eta_{L}: A \rightarrow A \amalg C$ to be the structure map $i_{1}$ of the coproduct, the right unit $\eta_{R}: A \rightarrow A \amalg C$ to be the coaction $\psi$, and the counit $\epsilon_{A}: A \amalg C \rightarrow A$ to be $1 \amalg \epsilon$. It is then obvious that $\epsilon_{A} \eta_{L}=1_{A}$, and $\epsilon_{A} \eta_{R}=1_{A}$ because $\psi$ is counital. We define the conjugation $\chi_{A}: A \amalg C \rightarrow A \amalg C$ to be $\psi$ on $A$ and $i_{2} \chi$ on $C$. Then $\chi_{A} \eta_{L}=\eta_{R}$ by definition, and $\chi_{A} \eta_{R}=\eta_{L}$ since $\chi^{2}=1$. Also, $\chi_{A}^{2}=1_{A \amalg C}$ for the same reasons. Finally, the diagonal $\Delta_{A}$ is the composite

$$
A \amalg C \stackrel{1 \amalg \Delta}{\longrightarrow} A \amalg C \amalg C \cong(A \amalg C) \amalg_{C}(A \amalg C),
$$

The fact that $\Delta$ is coassociative, counital, and compatible with $\chi$ implies the same facts for $\Delta_{A}$. 
We can now apply this to $(R, C(G, R))$,

Theorem 6.7. Suppose $G$ is a profinite group acting continuously on a ring $R$ that is complete in the a-adic topology for some ideal $\mathfrak{a}$. Then $(R, C(G, R))$ is a split formal Hopf algebroid.

This theorem is also true in the graded case, where the action of $G$ must preserve the grading, the ideal $\mathfrak{a}$ must be homogeneous, and $R$ need only be complete in the graded sense.

Proof. Think of $C(G, \mathbb{Z})$ as a cogroup object in the category of formal rings, where the topology is trivial. Recall from Proposition 2.5 that there is an isomorphism

$$
\sigma: R \widehat{\otimes} C(G, \mathbb{Z}) \rightarrow C(G, R) .
$$

Define a coaction $\psi$ of the cogroup $C(G, \mathbb{Z})$ on $R$ as the composite

$$
R \stackrel{\eta_{R}}{\longrightarrow} C(G, R) \stackrel{\sigma^{-1}}{\longrightarrow} R \widehat{\otimes} C(G, / Z),
$$

where $\eta_{R}(r)(g)=r^{g}$. It is easy to check that $\sigma$ is counital. To see that $\psi$ is coassociative, we use the following commutative diagram.

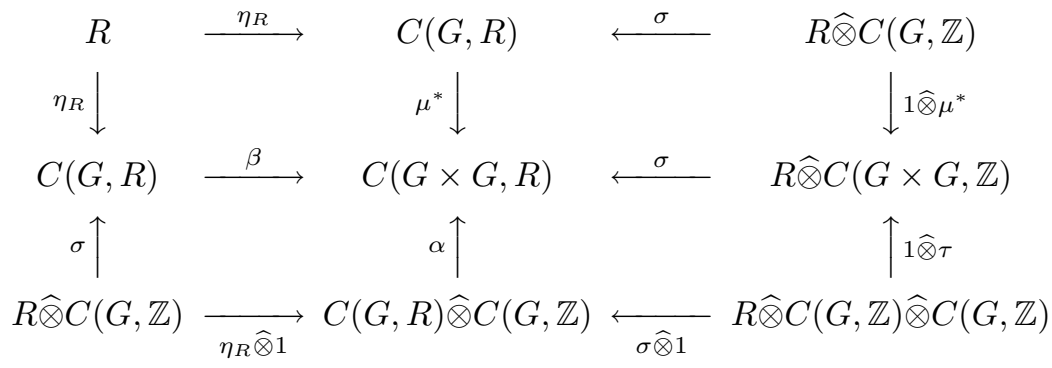

In this diagram, $\mu: G \times G \rightarrow G$ denotes the multiplication map, $\beta: C(G, R) \rightarrow$ $C(G \times G, R)$ is defined by $(\beta f)(g, h)=f(h)^{g}$, and $\alpha: C(G, R) \widehat{\otimes} C(G, \mathbb{Z}) \rightarrow C(G \times$ $G, R)$ is defined by $\alpha\left(f \otimes f^{\prime}\right)(g, h)=f^{\prime}(h) f(g)$. The reader can check that this diagram is in fact commutative. All the maps that go either left or up are isomorphisms, using Proposition 2.4 and the fact that $\tau$ is an isomorphism. Hence we can reverse those arrows, and then the equality of the outer composites shows that $\eta_{R}$ is coassociative. Hence Proposition 6.6 completes the proof.

We now describe the structure maps of $(R, C(G, R))$. The left unit is the inclusion of the constant functions, and the right unit $\eta_{R}$ is defined by $\eta_{R}(r)(g)=r^{g}$. The counit is evaluation at $e$, and the conjugation $\chi$ is defined by $(\chi f)(g)=f\left(g^{-1}\right)^{g}$. We have the commutative diagram below,

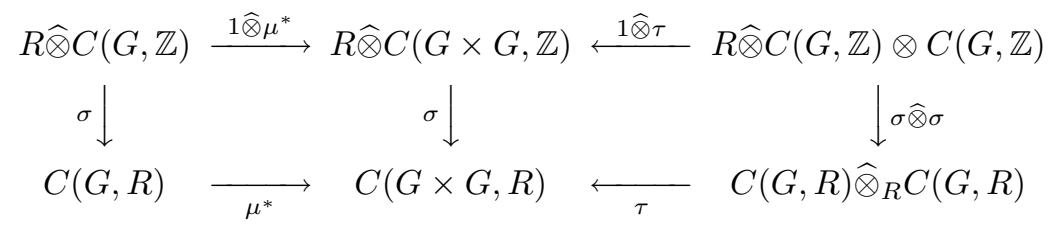


when the $\tau$ on the bottom line is defined to be the completion of the map defined by $\tau\left(f \otimes f^{\prime}\right)(g, h)=f(g) f^{\prime}(h)^{g}$. Hence $\tau$ is an isomorphism, and the diagonal is the composite of the bottom line after reversing $\tau$.

\section{4. $\Phi$ is a map of graded formal Hopf algebroids}

Theorem 6.8. The map

$$
(1, \Phi):\left(E_{*}, E_{*}^{\vee} E\right) \rightarrow\left(E_{*}, C\left(\Gamma, E_{*}\right)\right)
$$

is a map of graded formal Hopf algebroids.

Proof. we begin by showing that $\Phi \eta_{L}=\eta_{L}$. Indeed, if $a \in E_{m}$ and $\gamma \in \Gamma$, then we have

$$
\left(\Phi \eta_{L}(a)\right)(\gamma)=\mu \circ(1 \widetilde{\wedge} \gamma) \circ(1 \widetilde{\wedge} \eta) \circ a=\mu \circ(1 \widetilde{\wedge} \eta) \circ a=a=\left(\eta_{L}(a)(\gamma)\right.
$$

as required. Here we have used the fact that $\gamma \circ \eta=\eta$, since $\gamma$ is a map of ring spectra, Similarly, we have

$$
\left(\Phi \eta_{R}(a)\right)(\gamma)=\mu(1 \widetilde{\wedge} \gamma)(\eta \widetilde{\wedge} 1) a=\mu(\eta \widetilde{\wedge} 1) \gamma \circ a=\gamma \circ a=a^{\gamma}=\left(\eta_{R}(a)\right)(\gamma),
$$

To see that $\Phi$ commutes with $\epsilon$, we have

$$
\epsilon \Phi(a)=(\Phi a)(e)=\mu \circ(1 \widetilde{\wedge} e) \circ a=\mu \circ a=\epsilon(a) .
$$

And to see that $\Phi$ commutes with $\chi$ we compute:

$$
\begin{gathered}
{[\chi \Phi(a)](\gamma)=\left[\Phi(a)\left(\gamma^{-1}\right)\right]^{\gamma}=\gamma \circ \mu \circ\left(1 \widetilde{\wedge} \gamma^{-1}\right) \circ a} \\
=\mu \circ(\gamma \widetilde{\wedge} \gamma) \circ\left(1 \widetilde{\wedge} \gamma^{-1}\right) \circ a=\mu \circ(\gamma \widetilde{\wedge} 1) \circ a \\
=\mu \circ(1 \widetilde{\wedge} \gamma) \circ T \circ a=[\Phi(\chi a)](\gamma) .
\end{gathered}
$$

We are left with proving that $\Phi$ is compatible with $\Delta$ in the sense that $(\Phi \otimes$ $\Phi)_{\mathfrak{m}}^{\wedge} \circ \Delta=\Delta \circ \Phi$. We do this by constructing the commutative diagram below.

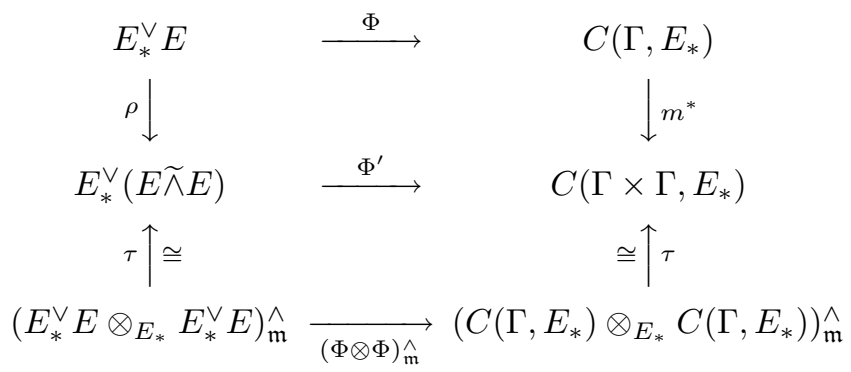

Here $\rho$ is induced by $1 \widetilde{\wedge} \eta \widetilde{\wedge} 1$, and of course the vertical composites (after inverting $\tau)$ are the diagonal maps. The map $\Phi^{\prime}$ will be defined analogously to $\Phi$. Indeed, we have a map

$$
\sigma^{\prime}: \Gamma \times \Gamma \times E_{*}^{\vee}(E \widetilde{\wedge} E) \rightarrow E_{*}
$$

that takes the triple $\left(\gamma, \gamma^{\prime}, a\right)$, where $a \in E_{m}^{\vee}(E \widetilde{\wedge} E)$ to the composite

$$
S^{m} \stackrel{a}{\rightarrow} E \widetilde{\wedge} E \widetilde{\wedge} E \stackrel{1 \widetilde{\wedge} \gamma \widetilde{\wedge} \gamma^{\prime}}{\longrightarrow} E \widetilde{\wedge} E \widetilde{\wedge} E \stackrel{1 \widetilde{\wedge} 1 \widetilde{\wedge} \gamma}{\longrightarrow} E \widetilde{\wedge} E \widetilde{\wedge} E \stackrel{1 \widetilde{\wedge} \mu}{\longrightarrow} E \widetilde{\wedge} E \stackrel{\mu}{\longrightarrow} E .
$$


Since $\sigma^{\prime}$ is a composite of composition maps and smash product maps, it will be continuous when every set of maps is given its natural topology (see the discussion at the beginning of Section 1). Thus the adjoint of $\sigma^{\prime}$ is our desired map

$$
\Phi^{\prime}: E_{*}^{\vee}(E \wedge E) \rightarrow C\left(\Gamma \times \Gamma, E_{*}\right) .
$$

Now we need to check that the two squares in our diagram (6.9) commute. For the top square, we have

$$
\begin{aligned}
\left(\Phi^{\prime} \rho(a)\right)\left(\gamma, \gamma^{\prime}\right) & =\mu \circ(1 \widetilde{\wedge} \mu) \circ(1 \widetilde{\wedge} 1 \widetilde{\wedge} \gamma) \circ\left(1 \widetilde{\wedge} \gamma \widetilde{\wedge} \gamma^{\prime}\right) \circ(1 \widetilde{\wedge} \eta \widetilde{\wedge} 1) \circ a \\
& =\mu \circ(1 \widetilde{\wedge} \mu) \circ(1 \widetilde{\wedge} 1 \widetilde{\wedge} \gamma) \circ(1 \widetilde{\wedge} \eta \widetilde{\wedge} 1) \circ\left(1 \widetilde{\wedge} \gamma^{\prime}\right) \circ a \\
& =\mu \circ(1 \widetilde{\wedge} \mu) \circ(1 \widetilde{\wedge} \eta \widetilde{\wedge} 1) \circ(1 \widetilde{\wedge} \gamma) \circ\left(1 \widetilde{\wedge} \gamma^{\prime}\right) \circ a \\
& =\mu \circ(1 \widetilde{\wedge} \gamma) \circ\left(1 \widetilde{\wedge} \gamma^{\prime}\right) \circ a \\
& =\left[m^{*} \Phi(a)\right]\left(\gamma, \gamma^{\prime}\right) .
\end{aligned}
$$

It suffices to check that the bottom square commutes before we complete the bottom row. We then have

$$
\begin{aligned}
{[\tau(\Phi \otimes \Phi)(a, b)]\left(\gamma, \gamma^{\prime}\right) } & =\mu \circ(1 \widetilde{\wedge} \gamma) \circ(\mu \widetilde{\wedge} \mu) \circ\left(1 \widetilde{\wedge} \gamma \widetilde{\wedge} 1 \widetilde{\wedge} \gamma^{\prime}\right) \circ(a \widetilde{\wedge} b) \\
& =\mu \circ(1 \widetilde{\wedge} \mu) \circ(1 \widetilde{\wedge} \gamma \widetilde{\wedge} \gamma) \circ(\mu \widetilde{\wedge} 1) \circ\left(1 \widetilde{\wedge} \gamma \widetilde{\wedge} 1 \widetilde{\wedge} \gamma^{\prime}\right) \circ(a \widetilde{\wedge} b) \\
& =\mu \circ(1 \widetilde{\wedge} \mu) \circ(\mu \widetilde{\wedge} 1 \widetilde{\wedge} 1) \circ\left(1 \widetilde{\wedge} \gamma \widetilde{\wedge} \gamma \widetilde{\wedge} \gamma \gamma^{\prime}\right) \circ(a \widetilde{\wedge} b) \\
& =\mu \circ(1 \widetilde{\wedge} \mu) \circ(1 \widetilde{\wedge} \mu \widetilde{\wedge} 1) \circ\left(1 \widetilde{\wedge} \gamma \widetilde{\wedge} \gamma \widetilde{\wedge} \gamma \gamma^{\prime}\right) \circ(a \widetilde{\wedge} b) \\
& =\mu \circ(1 \widetilde{\wedge} \mu) \circ\left(1 \widetilde{\wedge} \gamma \widetilde{\wedge} \gamma \gamma^{\prime}\right) \circ(1 \widetilde{\wedge} \mu \widetilde{\wedge} 1) \circ(a \widetilde{\wedge} b) \\
& =\left[\Phi^{\prime} \tau(a, b)\right]\left(\gamma, \gamma^{\prime}\right),
\end{aligned}
$$

completing the proof.

\section{Appendix A. The topology on $\Gamma$}

Recall that we identified the group $\Gamma$ in Theorem 4.9 as the semi-direct product $\Gamma_{0} \rtimes C$, where $\Gamma_{0}$ is the automorphism group of the Honda formal group law over $\mathbb{F}_{p^{n}}$ and $C$ is the Galois group of $\mathbb{F}_{p^{n}}$ over $\mathbb{F}_{p}$, This is an identification of abstract groups; for the isomorphism $\Phi: E_{*}^{\vee} E \rightarrow C\left(\Gamma, E_{*}\right)$ to be useful, we also need to understand the topology on $\Gamma$. At the moment, we know only that this topology is profinite. The topology on $\Gamma$ was described in [Str00], but many details are missing that we fill in here.

As explained in [Rav86, Lemma A2.2.16], the group $\Gamma_{0}$ is the group of units in the endomorphism ring $D$ of $F_{n}$, which is a noncommutative ring obtained from the Witt ring $W \mathbb{F}_{p^{n}}$ by adjoining an indeterminate $S$ subject to the relations $S^{n}=p$ and $S w=w^{\sigma} S$, where $\sigma$ denotes the generator of $C$. The group $C$ acts on $W \mathbb{F}_{p^{n}}$ according to [Rav86, Lemma A2.2.15]. The subgroup of $\Gamma_{0}$ consisting of the strict isomorphisms is called the Morava stabilizer group $S_{n}$ in [Rav86, Section 6.2].

We begin with some well-known facts about the stabilizer group $S_{n}$, whose proofs can be hard to find in the literature.

Lemma Appendix A.1. The Morava stabilizer group $S_{n}$ is a topologically finitely generated, pro-p group, and an open subgroup of finite index in $\Gamma_{0} \rtimes C$. 
Here a topological group is topologically finitely generated if it has a dense finitely generated subgroup. Note that it follows from Lemma Appendix A.1 that $\Gamma_{0} \rtimes C$, as a finite extension of $S_{n}$, is also topologically finitely generated.

Proof. First note that $D$ itself is a profinite ring. Indeed, by Lemma A2.2.16 of $[\operatorname{Rav86}], D$ is a free $\mathbb{Z}_{(p)}$-module of rank $n^{2}$, and since $p$ is central in $D$, we conclude that $D=\lim D /\left(p^{k}\right)$ as rings. It follows that $\Gamma_{0}$, as the group of units in $D$, is a profinite group (see the proof of Corollary 1.2). Hence $\Gamma_{0} \rtimes C$ is a profinite group as well in the product topology.

The stabilizer group $S_{n}$ is the preimage of 1 under the map of rings $D \rightarrow \mathbb{F}_{p^{n}}$ that takes an endomorphism of $F_{n}$ to the coefficient of $x$ in it. This map is the canonical reduction $\bmod p$ on $W$ and takes $S$ to 0 . Its kernel is the 2 -sided ideal $(S)$ generated by $S$, which is open since $S^{n}=p$. Hence this reduction map is continuous, so $S_{n}$ is closed. Since $\mathbb{F}_{p^{n}}$ is finite, $S_{n}$ has finite index in $D^{\times}$and so also in $\Gamma_{0} \rtimes C$, and so must also be open.

Since $S^{n}=p, D$ is complete in the $S$-adic topology. Hence $S_{n}$ is the inverse limit of the groups $G_{k}=\operatorname{ker}\left(D / S^{k}\right)^{\times} \rightarrow(D / S)^{\times}$. Since $S$ is nilpotent in $D / S^{k}$, this kernel is $1+H_{k}$, where $H_{k}=\operatorname{ker}\left(D / S^{k} \rightarrow D / S\right)$. In particular, $G_{k}$ is a finite $p$-group, so $S_{n}$ is a pro- $p$ group.

We now prove that $S_{n}$ is topologically finitely generated. Recall from [Rav86, Lemma A2.2.16] that every element of $S_{n}$ can be written as $1+\sum_{i=1}^{\infty} e_{i} S^{i}$, with $e_{i} \in \widetilde{\mathbb{F}_{p^{n}}}$, where $\widetilde{\mathbb{F}_{p^{n}}}$ is the set of all $e \in W \mathbb{F}_{p^{n}}$ such that $e^{p^{n}}=e$. The reduction map $W \mathbb{F}_{p^{n}} \rightarrow \mathbb{F}_{p^{n}}$ sends $\widetilde{\mathbb{F}_{p^{n}}}$ to $\mathbb{F}_{p^{n}}$ by a multiplicative bijection; $\widetilde{\mathbb{F}_{p^{n}}}$ is known as the set of Teichmuller lifts. Now, let

$$
T=\left\{1+e S^{k} \mid e \in \widetilde{\mathbb{F}_{p^{n}}}, 1 \leqslant k \leqslant \frac{n p}{p-1}\right\} .
$$

We claim that the subgroup generated by $T$ is dense. To see this, we first show that for all $e \in \widetilde{\mathbb{F}_{p^{n}}}$ and for all $k, T$ contains an element $x_{e, k}$ such that $x_{e, k} \cong 1+e S^{k}$ $\left(\bmod S^{k+1}\right)$. Indeed, this is obvious for all $k \leqslant \frac{n p}{p-1}$. For $k>\frac{n p}{p-1}$, we let $x_{e, k}=$ $x_{e, k-n}^{p}$. To see that $x_{e, k-n}^{p}$ has the required form, we use the fact that

$$
\begin{gathered}
\left(1+e S^{k-n}\right)^{p} \\
=1+p e S^{k-n}+\cdots+\left(\begin{array}{c}
p \\
i
\end{array}\right) e^{\left(p^{i}-1\right) /(p-1)} S^{i(k-n)}+\cdots+e^{\left(p^{n}-1\right) /(p-1)} S^{p(k-n)} \\
\cong 1+e S^{k}+e^{\left(p^{n}-1\right) /(p-1)} S^{p(k-n)} \quad\left(\bmod S^{k+1}\right),
\end{gathered}
$$

since $S^{n}=p$, and $S e=e^{p} S$ for $e \in \widetilde{\mathbb{F}_{p^{n}}}$. Now, since $k>\frac{n p}{p-1}$, we see that $p(k-n)>$ $k$, so $x_{e, k-n}^{p}$ is indeed a good choice for $x_{e, k}$.

Now, in order to see that $T$ is dense, it suffices to show that for all $k$ and $e_{1}, \ldots, e_{k} \in \widetilde{\mathbb{F}_{p^{n}}}, T$ contains an element congruent to $1+\sum_{i=1}^{k} e_{i} S^{i}$ modulo $S^{k+1}$. We prove this by induction on $k$, the base case being obvious. For the induction step, the induction hypothesis guarantees we can find an element

$$
y \cong 1+\sum_{i=1}^{k} e_{i} S^{i}+a S^{k+1} \quad\left(\bmod S^{k+2}\right)
$$


in $T$. We can also find an element $b \in \widetilde{\mathbb{F}_{p^{n}}}$ such that $a+b \cong e_{k+1}(\bmod p)$. Then

$$
\begin{gathered}
y x_{b, k+1} \cong 1+\sum_{i=1}^{k} e_{i} S^{i}+(a+b) S^{k+1} \quad\left(\bmod S^{k+2}\right) \\
\cong 1+\sum_{i=1}^{k} e_{i} S^{i}+e_{k+1} S^{k+1} \quad\left(\bmod S^{k+2}\right),
\end{gathered}
$$

as required, using the fact that $S^{n}=p$.

Theorem Appendix A.2. This isomorphism $\Gamma \cong \Gamma_{0} \rtimes C$ of Theorem 4.9 is a continuous isomorphism of profinite groups. Furthermore, a subgroup of $\Gamma$ is open if and only if it has finite index.

This theorem is saying that the topology on $\Gamma$ is completely determined by the group structure. This is believed to be true for a general profinite group, but remains an open question [CR02].

Proof. By [DdSMS99, Theorem 1.17], the open subgroups of a topologically finitely generated pro- $p$ group such as $S_{n}$ are precisely the subgroups of finite index. By [And76, Proposition 2], this remains true for any finite extension, such as $\Gamma_{0} \rtimes C$, of such a group. It follows that the isomorphism $\Gamma_{0} \rtimes C \rightarrow \Gamma$ of Theorem 4.9 is continuous. It is therefore a homeomorphism as well, since it is a map from a compact space to a Hausdorff space.

\section{References}

[Ada74] J. F. Adams, Stable homotopy and generalised homology, University of Chicago Press, Chicago, Ill., 1974, Chicago Lectures in Mathematics. MR 53 \#6534

[And76] Michael P. Anderson, Subgroups of finite index in profinite groups, Pacific J. Math. 62 (1976), no. 1, 19-28. MR 55 \#8182

[Bak89] Andrew Baker, Some families of operations in Morava K-theory, Amer. J. Math. 111 (1989), no. 1, 95-109. MR 90d:55030

[Bak95] A $\quad$ _ A version of Landweber's filtration theorem for $v_{n}$-periodic Hopf algebroids, Osaka J. Math. 32 (1995), no. 3, 689-699. MR 97h:55007

[CR02] Jon M. Corson and Thomas J. Ratkovich, On profinite groups whose power subgroups are closed, Comm. Algebra 30 (2002), no. 9, 41894196. MR 2003h:20055

[Dav04] Daniel Davis, The topological ring $E_{n *}^{s}\left[\left[\mathbb{G}_{n}\right]\right]$ and results on topological modules for Lubin-Tate theory, preprint, 2004.

[DdSMS99] J. D. Dixon, M. P. F. du Sautoy, A. Mann, and D. Segal, Analytic pro-p groups, second ed., Cambridge Studies in Advanced Mathematics, vol. 61, Cambridge University Press, Cambridge, 1999. MR 2000m:20039 
[DG70] Michel Demazure and Pierre Gabriel, Groupes algébriques. Tome I: Géométrie algébrique, généralités, groupes commutatifs, Masson \& Cie, Éditeur, Paris, 1970, Avec un appendice Corps de classes local par Michiel Hazewinkel. MR 46 \#1800

[DH04] Ethan S. Devinatz and Michael J. Hopkins, Homotopy fixed point spectra for closed subgroups of the Morava stabilizer groups, Topology 43 (2004), 1-47.

[EKMM97] A. D. Elmendorf, I. Kriz, M. A. Mandell, and J. P. May, Rings, modules, and algebras in stable homotopy theory, American Mathematical Society, Providence, RI, 1997, With an appendix by M. Cole. MR 97h:55006

[GHMR03] Paul Goerss, Hans-Werner Henn, Mark Mahowald, and Charles Rezk, A resolution of the $K_{2}$-local sphere, preprint, 2003.

[Hov04] Mark Hovey, Some spectral sequences in Morava E-theory, preprint, 2004.

[HPS97] Mark Hovey, John H. Palmieri, and Neil P. Strickland, Axiomatic stable homotopy theory, Mem. Amer. Math. Soc. 128 (1997), no. 610, $\mathrm{x}+114$. MR 98a:55017

[HR89] M. J. Hopkins and D. C. Ravenel, The Hopf algebroid E $(n)_{*} E(n)$, preprint, 1989

[HS99] Mark Hovey and Neil P. Strickland, Morava K-theories and localisation, Mem. Amer. Math. Soc. 139 (1999), no. 666, viii+100. MR 99b:55017

[HS03] Comodules and Landweber exact homology theories, preprint, 2003.

[Joh00] Keith Johnson, On the structure of the Hopf algebroid $E(n)_{*} E(n)$, Amer. J. Math. 122 (2000), no. 1, 223-234. MR 2001e:55008

[KMRT98] Max-Albert Knus, Alexander Merkurjev, Markus Rost, and JeanPierre Tignol, The book of involutions, American Mathematical Society Colloquium Publications, vol. 44, American Mathematical Society, Providence, RI, 1998, With a preface in French by J. Tits. MR 2000a:16031

[Lam99] T. Y. Lam, Lectures on modules and rings, Springer-Verlag, New York, 1999. MR 99i:16001

[LT66] Jonathan Lubin and John Tate, Formal moduli for one-parameter formal Lie groups, Bull. Soc. Math. France 94 (1966), 49-59. MR 39 \#214

[Mat89] Hideyuki Matsumura, Commutative ring theory, second ed., Cambridge Studies in Advanced Mathematics, vol. 8, Cambridge University Press, Cambridge, 1989, Translated from the Japanese by M. Reid. MR 90i:13001

[Mor85] Jack Morava, Noetherian localisations of categories of cobordism comodules, Ann. of Math. (2) 121 (1985), no. 1, 1-39. MR 86g:55004 
[Rav86] Douglas C. Ravenel, Complex cobordism and stable homotopy groups of spheres, Pure and Applied Mathematics, vol. 121, Academic Press Inc., Orlando, FL, 1986. MR 87j:55003

[Rez98] Charles Rezk, Notes on the Hopkins-Miller theorem, Homotopy theory via algebraic geometry and group representations (Evanston, IL, 1997), Contemp. Math., vol. 220, Amer. Math. Soc., Providence, RI, 1998, pp. 313-366. MR 2000i:55023

[SGA4] Théorie des topos et cohomologie étale des schémas. Tome 1: Théorie des topos, Springer-Verlag, Berlin, 1972, Séminaire de Géométrie Algébrique du Bois-Marie 1963-1964 (SGA 4), Dirigé par M. Artin, A. Grothendieck, et J. L. Verdier. Avec la collaboration de N. Bourbaki, P. Deligne et B. Saint-Donat, Lecture Notes in Mathematics, Vol. 269. MR 50 \#7130

[Str99] Neil P. Strickland, Formal schemes and formal groups, Homotopy invariant algebraic structures (Baltimore, MD, 1998), Amer. Math. Soc., Providence, RI, 1999, pp. 263-352. MR 2000j:55011

[Str00] N. P. Strickland, Gross-Hopkins duality, Topology 39 (2000), no. 5, 1021-1033. MR 2001d:55006

This article may be accessed via WWW at http://www.rmi.acnet.ge/hha/ or by anonymous ftp at

ftp://ftp.rmi.acnet.ge/pub/hha/volumes/2004/n1a13/v6n1a13.(dvi,ps,pdf)

Mark Hovey hovey@member.ams.org

Department of Mathematics

Wesleyan University

Middletown, CT 06459 